\title{
Integral identities for a semi-infinite interfacial crack in 2D and 3D elasticity
}

\author{
A. Piccolroaz*and G. Mishuris \\ Institute of Mathematical and Physical Sciences, Aberystwyth University, Wales, U.K.
}

September 11, 2018

\begin{abstract}
The paper is concerned with the problem of a semi-infinite crack at the interface between two dissimilar elastic half-spaces, loaded by a general asymmetrical system of forces distributed along the crack faces. On the basis of the weight function approach and the fundamental reciprocal identity (Betti formula), we formulate the elasticity problem in terms of singular integral equations relating the applied loading and the resulting crack opening. Such formulation is fundamental in the theory of elasticity and extensively used to solve several problems in linear elastic fracture mechanics (for instance various classic crack problems in homogeneous and heterogeneous media). This formulation is also crucial in important recent multiphysics applications, where the elastic problem is coupled with other concurrent physical phenomena. A paradigmatic example is hydraulic fracturing, where the elasticity equations are coupled with fluid dynamics.
\end{abstract}

Keywords: Interfacial crack; Singular integral; Betti identity; Weight function; Hydraulic fracture.

\section{Introduction}

Three-dimensional problems of linear elasticity can be solved nowadays by a variety of techniques. Purely numerical methods, such as finite element and boundary element techniques, are very popular and extensively used, thanks to the computational power of modern computers. However, analytical methods, such as integral transforms, variational inequalities, and singular integral equations, are still of great interest, for instance in deriving fundamental solutions for some basic geometries (to be employed in numerical methods), in the assertion of existence and uniqueness of the solution, and in bifurcation theory (Bigoni and Capuani, 2002; Bigoni and Dal Corso, 2008).

In particular, the method of singular integral equations was first developed for two-dimensional problems (Muskhelishvili, 1953) and later extended to three-dimensional problems by the rapidly developing theory of multi-dimensional singular integral operators (Kupradze et al., 1979; Mikhlin and Prossdörf, 1980). For crack problems, the general approach to derive the singular integral formulation is based on the Green's function method (Weaver, 1977; Budiansky and Rice, 1979, Linkov et al., 1997). This method consists in two steps. First, the stresses at a point $\boldsymbol{x}$ inside a body containing a discontinuity surface $S$ are expressed in the form

$$
\sigma_{i j}(\boldsymbol{x})=\int_{S} K_{i j k}(\boldsymbol{x}, \boldsymbol{\xi}) \Delta u_{k}(\boldsymbol{\xi}) d \boldsymbol{\xi},
$$

where $K_{i j k}(\boldsymbol{x}, \boldsymbol{\xi})=c_{i j s t}\left(\partial / \partial x_{t}\right) T_{s k}^{+}(\boldsymbol{x}, \boldsymbol{\xi})$ and $T_{s k}^{+}(\boldsymbol{x}, \boldsymbol{\xi})$ is the traction associated with the Green's function $U_{s p}(\boldsymbol{x}, \boldsymbol{\xi})$, according to the equation $T_{s k}^{+}(\boldsymbol{x}, \boldsymbol{\xi})=n_{l}^{+} c_{k l p q} U_{s p, q}(\boldsymbol{x}, \boldsymbol{\xi})$. Here $c_{i j s t}$ is the elasticity tensor, $n_{l}^{+}$is the outward normal on the + side of the surface and the displacement discontinuity is denoted by

\footnotetext{
*Corresponding author: e-mail: roaz@ing.unitn.it; phone: +390461 282583.
} 
$\Delta u_{k}=u_{k}^{-}-u_{k}^{+}$. The second step consists in taking the limit as the point $\boldsymbol{x}$ approaches a point $\boldsymbol{x}_{0}$ on the surface $S$, to obtain the tractions on the surface

$$
p_{i}^{+}\left(\boldsymbol{x}_{0}\right)=\lim _{\boldsymbol{x} \rightarrow \boldsymbol{x}_{0}} \int_{S} n_{j}^{+}(\boldsymbol{x}) K_{i j k}(\boldsymbol{x}, \boldsymbol{\xi}) \Delta u_{k}(\boldsymbol{\xi}) d \boldsymbol{\xi}
$$

The described procedure works when the tractions on the discontinuity surface $S$ are symmetrical. Moreover, the limit in (2) is not straightforward and due care should be taken to ensure the convergence of the integrals.

In this paper, we consider the three-dimensional problem of a semi-infinite crack at the interface between two dissimilar elastic half-spaces and apply a method which avoids the use of the Green's function and the tedious limiting procedure. We also do not use the assumption of symmetrical load.

Our results are based on integral transforms and two fundamental notions of linear elasticity: the Betti reciprocal theorem, on the one hand, and the weight function approach, on the other. The Betti identity has been extensively used in the perturbation analysis of two and three-dimensional cracks (Willis and Movchan, 1995; Bercial-Velez et al., 2005). The concept of weight function was introduced in linear fracture mechanics by Bueckner (1970) for two-dimensional problem and later extended to general three-dimensional problems by Willis and Movchan (1995), Piccolroaz et al. (2007).

We consider a semi-infinite crack at the interface between two dissimilar elastic half-spaces $\mathbb{R}_{ \pm}^{3}=$ $\left\{\boldsymbol{x}=\left(x_{1}, x_{2}, x_{3}\right) \in \mathbb{R}^{3}: \pm x_{2}>0\right\}$, see Fig. 1. The two elastic half-spaces are assumed to be isotropic with shear modulus and Poisson's ratio denoted by $\mu_{ \pm}$and $\nu_{ \pm}$, respectively. The crack lies on the halfplane $\mathbb{R}_{-}^{2}=\left\{\boldsymbol{x}=\left(x_{1}, x_{2}, x_{3}\right) \in \mathbb{R}^{3}: x_{1}<0, x_{2}=0\right\}$. The crack faces are loaded by a system of, not necessarily symmetrical, distributed forces $\boldsymbol{p}_{ \pm}$.

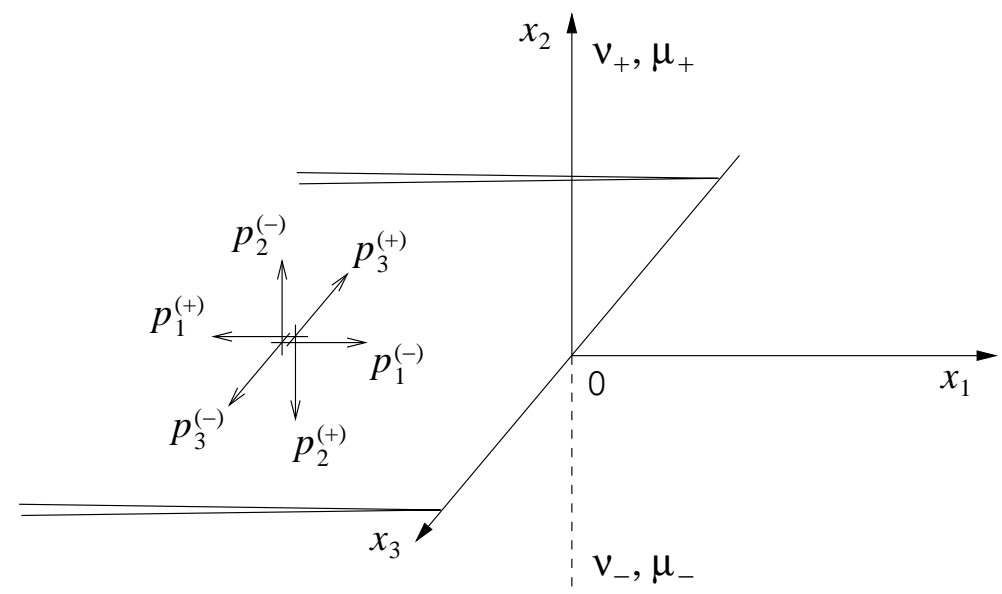

Figure 1: Semi-infinite interfacial crack loaded by asymmetrical forces on the crack faces.

It is convenient to introduce the symmetrical and skew-symmetrical parts of the loading as follows

$$
\langle\boldsymbol{p}\rangle=\frac{1}{2}\left(\boldsymbol{p}_{+}+\boldsymbol{p}_{-}\right), \quad \llbracket \boldsymbol{p} \rrbracket=\boldsymbol{p}_{+}-\boldsymbol{p}_{-},
$$

where we used standard notations to denote the average, $\langle f\rangle$, and the jump, $f f \rrbracket$, of a function $f$ across the plane containing the crack, $x_{2}=0$,

$$
\langle f\rangle\left(x_{1}, x_{3}\right)=\frac{1}{2}\left[f\left(x_{1}, 0^{+}, x_{3}\right)+f\left(x_{1}, 0^{-}, x_{3}\right)\right], \quad \llbracket f \rrbracket\left(x_{1}, x_{3}\right)=f\left(x_{1}, 0^{+}, x_{3}\right)-f\left(x_{1}, 0^{-}, x_{3}\right) .
$$

In Section 2, we introduce the fundamental reciprocal identity and the concept of weight function, as special singular solution to the homogeneous crack problem. These preliminary results are then used in the sequel for the analysis of both two and three-dimensional elastic problems. In particular, the two-dimensional problem is analysed in Section 3 (Mode III) and Section 4 (Mode I and II), while the general three-dimensional probelm is analysed in Section 5. 


\section{Preliminary Results. The Betti formula and weight functions}

In this section, we extend the Betti formula to the case of general asymmetrical loading applied at the crack surfaces. The Betti formula is used in order to relate the physical solution to the weight function, which is a special singular solution to the homogeneous problem (traction-free crack faces) (Willis and Movchan, 1995; Piccolroaz et al., 2007). For the sake of generality, we allow for traction and displacement jumps across the interface, so that results are general and applicable also to the case of imperfect interfaces.

In the absence of body forces, the Betti formula takes the form

$$
\int_{\partial \Omega}\left\{\boldsymbol{\sigma}^{(1)} \boldsymbol{n} \cdot \boldsymbol{u}^{(2)}-\boldsymbol{\sigma}^{(2)} \boldsymbol{n} \cdot \boldsymbol{u}^{(1)}\right\} d s=0,
$$

where $\partial \Omega$ is any surface enclosing a region $\Omega$ within which both displacement fields $\boldsymbol{u}^{(1)}$ and $\boldsymbol{u}^{(2)}$ satisfy the equations of equilibrium, with corresponding stress states $\boldsymbol{\sigma}^{(1)}$ and $\boldsymbol{\sigma}^{(2)}$, and $\boldsymbol{n}$ denotes the outward normal to $\partial \Omega$.

Applying the Betti formula to a hemispherical domain in the upper half-space $\mathbb{R}_{+}^{3}$, whose plane boundary is $x_{2}=0^{+}$and whose radius $R$ will be allowed to tend to infinity, we obtain, in the limit $R \rightarrow \infty$,

$$
\int_{\left(x_{2}=0^{+}\right)}\left\{\boldsymbol{\sigma}_{2}^{(1)}\left(x_{1}, 0^{+}, x_{3}\right) \cdot \boldsymbol{u}^{(2)}\left(x_{1}, 0^{+}, x_{3}\right)-\boldsymbol{\sigma}_{2}^{(2)}\left(x_{1}, 0^{+}, x_{3}\right) \cdot \boldsymbol{u}^{(1)}\left(x_{1}, 0^{+}, x_{3}\right)\right\} d x_{1} d x_{3}=0,
$$

provided that the fields $\boldsymbol{u}^{(1)}$ and $\boldsymbol{u}^{(2)}$ decay suitably fast at infinity. The notation $\boldsymbol{\sigma}_{2}$ is used to denote the traction vector acting on the plane $x_{2}=0: \boldsymbol{\sigma}_{2}=\boldsymbol{\sigma} \boldsymbol{e}_{2}$.

We can assume that $\boldsymbol{u}^{(1)}$ represents the physical field associated with the crack loaded at its surface, whereas $\boldsymbol{u}^{(2)}$ represents a non-trivial solution of the homogeneous problem, the so-called weight function, defined as follows

$$
\boldsymbol{u}^{(2)}\left(x_{1}, x_{2}, x_{3}\right)=\boldsymbol{R} \boldsymbol{U}\left(-x_{1}, x_{2},-x_{3}\right),
$$

where $\boldsymbol{R}$ is a rotation matrix

$$
\boldsymbol{R}=\left[\begin{array}{ccc}
-1 & 0 & 0 \\
0 & 1 & 0 \\
0 & 0 & -1
\end{array}\right]
$$

Note that the transformation (7) corresponds to introducing a change of coordinates in the solution $\boldsymbol{u}^{(2)}$, namely a rotation about the $x_{2}$-axis through an angle $\pi$. It is straightforward to verify that the weight function $\boldsymbol{U}$ satisfies the equations of equilibrium, but in a different domain where the crack is placed along the semi-plane $x_{2}=0, x_{1}>0$. The notation $\boldsymbol{\Sigma}$ will be used for components of stress corresponding to the displacement field $\boldsymbol{U}$,

$$
\boldsymbol{\sigma}^{(2)}\left(x_{1}, x_{2}, x_{3}\right)=\boldsymbol{R} \boldsymbol{\Sigma}\left(-x_{1}, x_{2},-x_{3}\right) \boldsymbol{R}
$$

Replacing $\boldsymbol{u}^{(2)}\left(x_{1}, x_{2}, x_{3}\right)$ with $\boldsymbol{u}^{(2)}\left(x_{1}-x_{1}^{\prime}, x_{2}, x_{3}-x_{3}^{\prime}\right)$, which corresponds to a shift within the plane $\left(x_{1}, x_{3}\right)$, we obtain

$\int_{\left(x_{2}=0^{+}\right)}\left\{\boldsymbol{R U}\left(x_{1}^{\prime}-x_{1}, 0^{+}, x_{3}^{\prime}-x_{3}\right) \cdot \boldsymbol{\sigma}_{2}\left(x_{1}, 0^{+}, x_{3}\right)-\boldsymbol{R} \boldsymbol{\Sigma}_{2}\left(x_{1}^{\prime}-x_{1}, 0^{+}, x_{3}^{\prime}-x_{3}\right) \cdot \boldsymbol{u}\left(x_{1}, 0^{+}, x_{3}\right)\right\} d x_{1} d x_{3}=0$.

A similar equation can be derived by applying the Betti formula to a hemispherical domain in the lower half-space $\mathbb{R}_{-}^{3}$,

$\int_{\left(x_{2}=0^{-}\right)}\left\{\boldsymbol{R U}\left(x_{1}^{\prime}-x_{1}, 0^{-}, x_{3}^{\prime}-x_{3}\right) \cdot \boldsymbol{\sigma}_{2}\left(x_{1}, 0^{-}, x_{3}\right)-\boldsymbol{R} \boldsymbol{\Sigma}_{2}\left(x_{1}^{\prime}-x_{1}, 0^{-}, x_{3}^{\prime}-x_{3}\right) \cdot \boldsymbol{u}\left(x_{1}, 0^{-}, x_{3}\right)\right\} d x_{1} d x_{3}=0$. 
Subtracting (11) from (10), we obtain

$$
\begin{array}{r}
\int_{\left(x_{2}=0\right)}\left\{\boldsymbol{R} \llbracket \boldsymbol{U} \rrbracket\left(x_{1}^{\prime}-x_{1}, x_{3}^{\prime}-x_{3}\right) \cdot\left\langle\boldsymbol{\sigma}_{2}\right\rangle\left(x_{1}, x_{3}\right)+\boldsymbol{R}\langle\boldsymbol{U}\rangle\left(x_{1}^{\prime}-x_{1}, x_{3}^{\prime}-x_{3}\right) \cdot \llbracket \boldsymbol{\sigma}_{2} \rrbracket\left(x_{1}, x_{3}\right)-\right. \\
\left.-\boldsymbol{R}\left\langle\boldsymbol{\Sigma}_{2}\right\rangle\left(x_{1}^{\prime}-x_{1}, x_{3}^{\prime}-x_{3}\right) \cdot \llbracket \boldsymbol{u} \rrbracket\left(x_{1}, x_{3}\right)\right\} d x_{1} d x_{3}=0 .
\end{array}
$$

Let us introduce the notations

$$
f^{(+)}\left(x_{1}, x_{3}\right)=f\left(x_{1}, x_{3}\right) H\left(x_{1}\right), \quad f^{(-)}\left(x_{1}, x_{3}\right)=f\left(x_{1}, x_{3}\right) H\left(-x_{1}\right),
$$

where $H$ denotes the Heaviside function, so that

$$
f\left(x_{1}, x_{3}\right)=f^{(+)}\left(x_{1}, x_{3}\right)+f^{(-)}\left(x_{1}, x_{3}\right) .
$$

The reciprocity identity (12) becomes

$$
\begin{aligned}
& \int_{-\infty}^{\infty}\left\{\boldsymbol{R} \llbracket \boldsymbol{U} \rrbracket\left(x_{1}^{\prime}-x_{1}, x_{3}^{\prime}-x_{3}\right) \cdot\left\langle\boldsymbol{\sigma}_{2}\right\rangle^{(+)}\left(x_{1}, x_{3}\right)-\boldsymbol{R}\left\langle\boldsymbol{\Sigma}_{2}\right\rangle\left(x_{1}^{\prime}-x_{1}, x_{3}^{\prime}-x_{3}\right) \cdot \llbracket \boldsymbol{u} \rrbracket^{(-)}\left(x_{1}, x_{3}\right)\right\} d x_{1} d x_{3}= \\
& -\int_{-\infty}^{\infty}\left\{\boldsymbol{R} \llbracket \boldsymbol{U} \rrbracket\left(x_{1}^{\prime}-x_{1}, x_{3}^{\prime}-x_{3}\right) \cdot\left\langle\boldsymbol{\sigma}_{2}\right\rangle^{(-)}\left(x_{1}, x_{3}\right)+\boldsymbol{R}\langle\boldsymbol{U}\rangle\left(x_{1}^{\prime}-x_{1}, x_{3}^{\prime}-x_{3}\right) \cdot \llbracket \boldsymbol{\sigma}_{2} \rrbracket^{(-)}\left(x_{1}, x_{3}\right)\right\} d x_{1} d x_{3} \\
& -\int_{-\infty}^{\infty}\left\{\boldsymbol{R}\langle\boldsymbol{U}\rangle\left(x_{1}^{\prime}-x_{1}, x_{3}^{\prime}-x_{3}\right) \cdot \llbracket \boldsymbol{\sigma}_{2} \rrbracket^{(+)}\left(x_{1}, x_{3}\right)-\boldsymbol{R}\left\langle\boldsymbol{\Sigma}_{2}\right\rangle\left(x_{1}^{\prime}-x_{1}, x_{3}^{\prime}-x_{3}\right) \cdot \llbracket \boldsymbol{u} \rrbracket^{(+)}\left(x_{1}, x_{3}\right)\right\} d x_{1} d x_{3} .
\end{aligned}
$$

The identity (14) can be written in an equivalent form using the convolution with respect to both $x_{1}$ and $x_{3}$ variables, denoted by the symbol $\circledast$,

$$
\boldsymbol{R} \llbracket \boldsymbol{U} \rrbracket \circledast\left\langle\boldsymbol{\sigma}_{2}\right\rangle^{(+)}-\boldsymbol{R}\left\langle\boldsymbol{\Sigma}_{2}\right\rangle \circledast \llbracket \boldsymbol{u} \rrbracket^{(-)}=-\boldsymbol{R} \llbracket \boldsymbol{U} \rrbracket \circledast\left\langle\boldsymbol{\sigma}_{2}\right\rangle^{(-)}-\boldsymbol{R}\langle\boldsymbol{U}\rangle \circledast \llbracket \boldsymbol{\sigma}_{2} \rrbracket^{(-)}-\boldsymbol{R}\langle\boldsymbol{U}\rangle \circledast \llbracket \boldsymbol{\sigma}_{2} \rrbracket^{(+)}+\boldsymbol{R}\left\langle\boldsymbol{\Sigma}_{2}\right\rangle \circledast \llbracket \boldsymbol{u} \rrbracket^{(+)} .
$$

Note that the superscripts ${ }^{(+)}$and ${ }^{(-)}$denote functions whose support is restricted to the positive and negative semi-axes, respectively. Thus, $\left\langle\boldsymbol{\sigma}_{2}\right\rangle^{(+)}$is the traction along the interface, ahead of the crack tip, whereas $\llbracket \boldsymbol{u} \rrbracket^{(-)}$is the crack opening (displacement discontinuity across the crack faces).

In the sequel, we will assume perfect contact conditions at the interface, so that traction and displacement are continuous for $x_{1}>0: \llbracket \boldsymbol{\sigma}_{2} \rrbracket^{(+)}=\llbracket \boldsymbol{u} \rrbracket^{(+)}=0$. Finally, the symmetric and skew-symmetric loads applied on the crack faces will be denoted by $\left\langle\boldsymbol{\sigma}_{2}\right\rangle^{(-)}=\langle\boldsymbol{p}\rangle$ and $\llbracket \boldsymbol{\sigma}_{2} \rrbracket^{(-)}=\llbracket \boldsymbol{p} \rrbracket$, respectively.

The terms $\llbracket \boldsymbol{U} \rrbracket$ and $\langle\boldsymbol{U}\rangle$ are known as the symmetric and skew-symmetric weight functions. The notion of skew-symmetric weight function was introduced in Piccolroaz et al. (2009) for problems of cracks loaded by asymmetrical forces. The term $\left\langle\boldsymbol{\Sigma}_{2}\right\rangle$ stands for the traction along the plane $x_{2}=0$, corresponding to the singular solution $\boldsymbol{U}$.

\section{Mode III}

In the case of antiplane deformation, the Betti formula (15) relating the physical field $u_{3}, \sigma_{23}$ with the weight function $U_{3}, \Sigma_{23}$ reduces to the scalar equation

$$
\llbracket U_{3} \rrbracket *\left\langle\sigma_{23}\right\rangle^{(+)}-\left\langle\Sigma_{23}\right\rangle * \llbracket u_{3} \rrbracket^{(-)}=-\llbracket U_{3} \rrbracket *\left\langle p_{3}\right\rangle-\left\langle U_{3}\right\rangle * \llbracket p_{3} \rrbracket,
$$

where the symbol $*$ denotes the convolution with respect to the variable $x_{1}$.

Let us introduce the Fourier transform with respect to the variable $x_{1}$ as follows

$$
\tilde{f}(\beta)=\mathcal{F}\left[f\left(x_{1}\right)\right]=\int_{-\infty}^{\infty} f\left(x_{1}\right) e^{i \beta x_{1}} d x_{1} .
$$

Applying the Fourier transform to the identity (16) we get

$$
\llbracket \tilde{U}_{3} \rrbracket\left\langle\tilde{\sigma}_{23}\right\rangle^{+}-\left\langle\tilde{\Sigma}_{23}\right\rangle \llbracket \tilde{u}_{3} \rrbracket^{-}=-\llbracket \tilde{U}_{3} \rrbracket\left\langle\tilde{p}_{3}\right\rangle-\left\langle\tilde{U}_{3}\right\rangle \llbracket \tilde{p}_{3} \rrbracket,
$$


where the superscripts ${ }^{+}$and ${ }^{-}$denote functions analytic in the upper and lower half-planes, respectively.

We can now multiply both sides of (18) by $\llbracket \tilde{U}_{3} \rrbracket^{-1}$ to obtain

$$
\left\langle\tilde{\sigma}_{23}\right\rangle^{+}-B \llbracket \tilde{u}_{3} \rrbracket^{-}=-\left\langle\tilde{p}_{3}\right\rangle-A \llbracket \tilde{p}_{3} \rrbracket,
$$

where the factors in front of unknown functions are given by

$$
A=\llbracket \tilde{U}_{3} \rrbracket^{-1}\left\langle\tilde{U}_{3}\right\rangle, \quad B=\llbracket \tilde{U}_{3} \rrbracket^{-1}\left\langle\tilde{\Sigma}_{23}\right\rangle .
$$

They can be computed from the relationships, which hold for the symmetric and skew-symmetric weight functions (Piccolroaz et al., 2009)

$$
\llbracket \tilde{U}_{3} \rrbracket=-\frac{b+e}{|\beta|}\left\langle\tilde{\Sigma}_{23}\right\rangle, \quad\left\langle\tilde{U}_{3}\right\rangle=\frac{\eta}{2} \llbracket \tilde{U}_{3} \rrbracket .
$$

Thus, we can easily obtain

$$
A=\frac{\eta}{2}, \quad B=-\frac{|\beta|}{b+e},
$$

where $b, e$ and $\eta$ are the following bimaterial constants,

$$
b=\frac{1-\nu_{+}}{\mu_{+}}+\frac{1-\nu_{-}}{\mu_{-}}, \quad e=\frac{\nu_{+}}{\mu_{+}}+\frac{\nu_{-}}{\mu_{-}}, \quad \eta=\frac{\mu_{-}-\mu_{+}}{\mu_{-}+\mu_{+}} .
$$

If we apply the inverse Fourier transform to (19), we obtain for the two opposite cases $x_{1}<0$ and $x_{1}>0$ the following relationships:

$$
\begin{gathered}
\left\langle p_{3}\right\rangle+\mathcal{F}_{x_{1}<0}^{-1}\left[A \llbracket \tilde{p}_{3} \rrbracket\right]=\mathcal{F}_{x_{1}<0}^{-1}\left[B \llbracket \tilde{u}_{3} \rrbracket^{-}\right], \\
\left\langle\sigma_{23}\right\rangle^{(+)}=\mathcal{F}_{x_{1}>0}^{-1}\left[B \llbracket \tilde{u}_{3} \rrbracket^{-}\right],
\end{gathered}
$$

Note that the term $\left\langle\tilde{\sigma}_{23}\right\rangle^{+}$in (18) cancels from (24) because it is a "+" function, while the terms $\left\langle\tilde{p}_{3}\right\rangle$ and $\llbracket \tilde{p}_{3} \rrbracket$ cancel from (25) because they are "-" functions.

Finally, we find the inverse Fourier transform of the function $|\beta| \llbracket \tilde{u}_{3} \rrbracket^{-}$. To this purpose we write $|\beta| \llbracket \tilde{u}_{3} \rrbracket^{-}=\operatorname{sign}(\beta) \cdot \beta \llbracket \tilde{u}_{3} \rrbracket^{-}$and observe that $\operatorname{sign}(\beta)$ is the Fourier transform of $-i /\left(\pi x_{1}\right)$, whereas $\beta \llbracket \tilde{u}_{3} \rrbracket^{-}$is the Fourier transform of $i \partial \llbracket u_{3} \rrbracket^{-} / \partial x_{1}$. Therefore we get

$$
\mathcal{F}^{-1}\left[|\beta| \llbracket \tilde{u}_{3} \rrbracket^{-}\right]=\frac{1}{\pi x_{1}} * \frac{\partial \llbracket u_{3} \rrbracket^{-}}{\partial x_{1}} .
$$

Note that, with slight abuse of notation, we write the convolution of two functions $f\left(x_{1}\right)$ and $g\left(x_{1}\right)$ in the form

$$
\int_{-\infty}^{\infty} f\left(x_{1}-\xi\right) g(\xi) d \xi=f\left(x_{1}\right) * g\left(x_{1}\right),
$$

so that the right-hand side in (26) has to be interpreted as follows

$$
\frac{1}{\pi x_{1}} * \frac{\partial \llbracket u_{3} \rrbracket^{-}}{\partial x_{1}}=\frac{1}{\pi} \int_{-\infty}^{\infty} \frac{1}{x_{1}-\xi} \frac{\partial \llbracket u_{3} \rrbracket^{-}}{\partial \xi} d \xi .
$$

To simplify notations, we introduce the singular operator $\mathcal{S}$ and the orthogonal projectors $\mathcal{P}_{ \pm}\left(\mathcal{P}_{+}+\mathcal{P}_{-}=\right.$ $\mathcal{I})$ acting on the real axis:

$$
\begin{gathered}
\psi=\mathcal{S} \varphi=\frac{1}{\pi x_{1}} * \varphi\left(x_{1}\right)=\frac{1}{\pi} \int_{-\infty}^{\infty} \frac{\varphi(\xi)}{x_{1}-\xi} d \xi \\
\mathcal{P}_{ \pm} \varphi= \begin{cases}\varphi\left(x_{1}\right) & \pm x_{1} \geq 0 \\
0 & \text { otherwise }\end{cases}
\end{gathered}
$$


Note that the operator $\mathcal{S}$ is the classic singular operator of the Cauchy type; it transforms any function $\varphi$, satisfying the Hölder condition, into a new function $\mathcal{S} \varphi$ which also satisfies the Hölder condition (Muskhelishvili, 1946). The properties of the operator $\mathcal{S}$ in other functional spaces have been discussed in Prössdorf (1974).

The integral identities (24) and (25) for Mode III deformation become

$$
\begin{gathered}
\left\langle p_{3}\right\rangle+\frac{\eta}{2} \llbracket p_{3} \rrbracket=-\frac{1}{b+e} \mathcal{S}^{(s)} \frac{\partial \llbracket u_{3} \rrbracket^{(-)}}{\partial x_{1}}, \quad x_{1}<0, \\
\left\langle\sigma_{23}\right\rangle^{(+)}=-\frac{1}{b+e} \mathcal{S}^{(c)} \frac{\partial \llbracket u_{3} \rrbracket^{(-)}}{\partial x_{1}}, \quad x_{1}>0,
\end{gathered}
$$

where $\mathcal{S}^{(s)}=\mathcal{P}_{-} \mathcal{S} \mathcal{P}_{-}$is a singular operator, whereas $\mathcal{S}^{(c)}=\mathcal{P}_{+} \mathcal{S} \mathcal{P}_{-}$is a compact operator (see Gakhov and Cherski 1978; Krein, 1958; Gohberg and Krein, 1958). Despite the fact that these operators look similar at a first glance, they are essentially different. Indeed, $\mathcal{S}^{(s)}: F\left(\mathbb{R}_{-}\right) \rightarrow F\left(\mathbb{R}_{-}\right)$, while $\mathcal{S}^{(c)}$ : $F\left(\mathbb{R}_{-}\right) \rightarrow F\left(\mathbb{R}_{+}\right)$, where $F\left(\mathbb{R}_{ \pm}\right)$is some functional space of functions defined on $\mathbb{R}_{ \pm}$.

To make this point clear, let us write eqs. (31) and (32) in extended form as

$$
\begin{gathered}
\left\langle p_{3}\right\rangle+\frac{\eta}{2} \llbracket p_{3} \rrbracket=-\frac{1}{\pi(b+e)} \int_{-\infty}^{0} \frac{1}{x_{1}-\xi} \frac{\partial \llbracket u_{3} \rrbracket^{(-)}}{\partial \xi} d \xi, \quad x_{1}<0, \\
\left\langle\sigma_{23}\right\rangle^{(+)}=-\frac{1}{\pi(b+e)} \int_{-\infty}^{0} \frac{1}{x_{1}-\xi} \frac{\partial \llbracket u_{3} \rrbracket^{(-)}}{\partial \xi} d \xi, \quad x_{1}>0 .
\end{gathered}
$$

The integral in (33) is a Cauchy singular integral with a moving singularity, whereas the integral in (34) is an integral with a fixed point singularity.

Note that, in the particular case of a homogeneous body, we have $\eta=0$ and $b+e=2 / \mu$, so that the integral identities (31) and (32) reduce to

$$
\begin{gathered}
\left\langle p_{3}\right\rangle=-\frac{\mu}{2} \mathcal{S}^{(s)} \frac{\partial \llbracket u_{3} \rrbracket^{(-)}}{\partial x_{1}}, \quad x_{1}<0, \\
\left\langle\sigma_{23}\right\rangle^{(+)}=-\frac{\mu}{2} \mathcal{S}^{(c)} \frac{\partial \llbracket u_{3} \rrbracket^{(-)}}{\partial x_{1}}, \quad x_{1}>0 .
\end{gathered}
$$

The two equations (33) and (34) form the system of integral identities for the Mode III deformation. The first equation (33) provides the integral relationship between the applied loading $\left\langle p_{3}\right\rangle, \llbracket p_{3} \rrbracket$ and the resulting crack opening $\llbracket u_{3} \rrbracket^{(-)}$. This is a singular integral equation and it is, generally speaking, invertible. However, the inverse operator depends on the properties of the solution. The second equation (34) can be considered as an additional equation which allows to define the proper behaviour of the solution $\llbracket u_{3} \rrbracket^{(-)}$and also, after the first equation has been inverted, to evaluate the traction ahead of the crack tip $\left\langle\sigma_{23}\right\rangle^{(+)}$. The reason for this is that the operator in the right-hand side of (34) is a compact one and thus it is not invertible.

More details on the theory of singular integral equations can be found in Muskhelishvili (1946). The inversion of the singular operator $\mathcal{S}^{(s)}$, in some specific cases of interest for fracture mechanics and, in particular, for hydraulic fracturing, is discussed in Appendix A.

\section{Mode I and II}

In the case of plane strain deformation, the Betti identity (15) relating the physical solution $\boldsymbol{u}=\left[u_{1}, u_{2}\right]^{T}$, $\boldsymbol{\sigma}_{2}=\left[\sigma_{21}, \sigma_{22}\right]^{T}$ with the weight function $\boldsymbol{U}, \boldsymbol{\Sigma}_{2}$ is given by

$$
\boldsymbol{R} \llbracket \boldsymbol{U} \rrbracket *\left\langle\boldsymbol{\sigma}_{2}\right\rangle^{(+)}-\boldsymbol{R}\left\langle\boldsymbol{\Sigma}_{2}\right\rangle * \llbracket \boldsymbol{u} \rrbracket^{(-)}=-\boldsymbol{R} \llbracket \boldsymbol{U} \rrbracket *\langle\boldsymbol{p}\rangle-\boldsymbol{R}\langle\boldsymbol{U}\rangle * \llbracket \boldsymbol{p} \rrbracket .
$$


where $\langle\boldsymbol{p}\rangle=\left[\left\langle p_{1}\right\rangle,\left\langle p_{2}\right\rangle\right]^{T}, \llbracket \boldsymbol{p} \rrbracket=\left[\llbracket p_{1} \rrbracket, \llbracket p_{2} \rrbracket\right]^{T}$ are the symmetric and skew-symmetric parts of the loading. Again we assume ideal contact conditions, so that traction and displacement are continuous across the interface: $\llbracket \boldsymbol{\sigma}_{2} \rrbracket^{(+)}=\llbracket \boldsymbol{u} \rrbracket^{(+)}=0$. Here and in the sequel of this section, we use the following matrices:

$$
\boldsymbol{R}=\left[\begin{array}{cc}
-1 & 0 \\
0 & 1
\end{array}\right], \quad \boldsymbol{I}=\left[\begin{array}{ll}
1 & 0 \\
0 & 1
\end{array}\right], \quad \boldsymbol{E}=\left[\begin{array}{cc}
0 & 1 \\
-1 & 0
\end{array}\right]
$$

Note that the symmetric and skew-symmetric weight functions, $\llbracket \boldsymbol{U} \rrbracket$ and $\langle\boldsymbol{U}\rangle$, and the corresponding traction $\left\langle\boldsymbol{\Sigma}_{2}\right\rangle$ are represented by $2 \times 2$ matrices. In fact, in the case of an elastic bimaterial plane, there are two linearly independent weight functions, $\boldsymbol{U}^{j}=\left[U_{1}^{j}, U_{2}^{j}\right]^{T}, \boldsymbol{\Sigma}_{2}^{j}=\left[\Sigma_{21}^{j}, \Sigma_{22}^{j}\right]^{T}, j=1,2$, and it is possible to construct the weight function tensors by ordering the components of each weight function in columns of $2 \times 2$ matrices (Piccolroaz et al., 2009)

$$
\boldsymbol{U}=\left[\begin{array}{cc}
U_{1}^{1} & U_{1}^{2} \\
U_{2}^{1} & U_{2}^{2}
\end{array}\right], \quad \boldsymbol{\Sigma}_{2}=\left[\begin{array}{cc}
\Sigma_{21}^{1} & \Sigma_{21}^{2} \\
\Sigma_{22}^{1} & \Sigma_{22}^{2}
\end{array}\right]
$$

Applying the Fourier transform to the equation (37), we obtain

$$
\llbracket \tilde{\boldsymbol{U}} \rrbracket^{T} \boldsymbol{R}\left\langle\tilde{\boldsymbol{\sigma}}_{2}\right\rangle^{+}-\left\langle\tilde{\boldsymbol{\Sigma}}_{2}\right\rangle^{T} \boldsymbol{R} \llbracket \tilde{\boldsymbol{u}} \rrbracket^{-}=-\llbracket \tilde{\boldsymbol{U}} \rrbracket^{T} \boldsymbol{R}\langle\tilde{\boldsymbol{p}}\rangle-\langle\tilde{\boldsymbol{U}}\rangle^{T} \boldsymbol{R} \llbracket \tilde{\boldsymbol{p}} \rrbracket .
$$

Multiplying both sides by $\boldsymbol{R}^{-1} \llbracket \tilde{\boldsymbol{U}} \rrbracket^{-T}$ we get

$$
\left\langle\tilde{\boldsymbol{\sigma}}_{2}\right\rangle^{+}-\boldsymbol{B} \llbracket \tilde{\boldsymbol{u}} \rrbracket^{-}=-\langle\tilde{\boldsymbol{p}}\rangle-\boldsymbol{A} \llbracket \tilde{\boldsymbol{p}} \rrbracket,
$$

where $\boldsymbol{A}$ and $\boldsymbol{B}$ are the following matrices

$$
\boldsymbol{A}=\boldsymbol{R}^{-1} \llbracket \tilde{\boldsymbol{U}} \rrbracket^{-T}\langle\tilde{\boldsymbol{U}}\rangle^{T} \boldsymbol{R}, \quad \boldsymbol{B}=\boldsymbol{R}^{-1} \llbracket \tilde{\boldsymbol{U}} \rrbracket^{-T}\left\langle\tilde{\boldsymbol{\Sigma}}_{2}\right\rangle^{T} \boldsymbol{R},
$$

which can be computed using results for the symmetric and skew-symmetric weight functions obtained in Antipov (1999) and Piccolroaz et al. (2009). Namely

$$
\llbracket \tilde{\boldsymbol{U}} \rrbracket=-\frac{1}{|\beta|}[b \boldsymbol{I}-i d \operatorname{sign}(\beta) \boldsymbol{E}]\left\langle\tilde{\boldsymbol{\Sigma}}_{2}\right\rangle, \quad\langle\tilde{\boldsymbol{U}}\rangle=-\frac{b}{2|\beta|}[\alpha \boldsymbol{I}-i \gamma \operatorname{sign}(\beta) \boldsymbol{E}]\left\langle\tilde{\boldsymbol{\Sigma}}_{2}\right\rangle,
$$

where $b$ is defined in (23),$d / b$ and $\alpha$ are the so-called Dundurs parameters, while $\gamma$ is another bimaterial constant:

$$
\begin{gathered}
d=\frac{1-2 \nu_{+}}{2 \mu_{+}}-\frac{1-2 \nu_{-}}{2 \mu_{-}} \\
\alpha=\frac{\mu_{-}\left(1-\nu_{+}\right)-\mu_{+}\left(1-\nu_{-}\right)}{\mu_{-}\left(1-\nu_{+}\right)+\mu_{+}\left(1-\nu_{-}\right)}, \quad \gamma=\frac{\mu_{-}\left(1-2 \nu_{+}\right)+\mu_{+}\left(1-2 \nu_{-}\right)}{2 \mu_{-}\left(1-\nu_{+}\right)+2 \mu_{+}\left(1-\nu_{-}\right)} .
\end{gathered}
$$

As a result, we obtain

$$
\begin{gathered}
\boldsymbol{A}=\frac{b}{2\left(b^{2}-d^{2}\right)}[(b \alpha-d \gamma) \boldsymbol{I}+i(d \alpha-b \gamma) \operatorname{sign}(\beta) \boldsymbol{E}], \\
\boldsymbol{B}=-\frac{|\beta|}{b^{2}-d^{2}}[b \boldsymbol{I}+i d \operatorname{sign}(\beta) \boldsymbol{E}] .
\end{gathered}
$$

Inverting the Fourier transform for the two cases $x_{1}<0$ and $x_{1}>0$, we get

$$
\begin{gathered}
\langle\boldsymbol{p}\rangle+\mathcal{F}_{x_{1}<0}^{-1}[\boldsymbol{A} \llbracket \tilde{\boldsymbol{p}} \rrbracket]=\mathcal{F}_{x_{1}<0}^{-1}\left[\boldsymbol{B} \llbracket \tilde{\boldsymbol{u}} \rrbracket^{-}\right], \\
\left\langle\boldsymbol{\sigma}_{2}\right\rangle^{(+)}+\mathcal{F}_{x_{1}>0}^{-1}[\boldsymbol{A} \llbracket \tilde{\boldsymbol{p}} \rrbracket]=\mathcal{F}_{x_{1}>0}^{-1}\left[\boldsymbol{B} \llbracket \tilde{\boldsymbol{u}} \rrbracket^{-}\right] .
\end{gathered}
$$

Similarly to the previous section, the term $\langle\tilde{\boldsymbol{\sigma}}\rangle^{+}$in (40) cancels from (48) because it is a "+" function, whereas the term $\langle\tilde{\boldsymbol{p}}\rangle$ cancels from (49) because it is a "-" function. 
To proceed further, we need to invert functions of the form $\operatorname{sign}(\beta) \llbracket \tilde{p}_{i} \rrbracket,|\beta| \llbracket \tilde{u}_{i} \rrbracket$ and $\beta \llbracket \tilde{u}_{i} \rrbracket$. This is done in a way similar to the previous section and we finally obtain the integral identities for plane strain deformation as follows

$$
\begin{gathered}
\langle\boldsymbol{p}\rangle+\mathcal{A}^{(s)} \llbracket \boldsymbol{p} \rrbracket=\mathcal{B}^{(s)} \frac{\partial \llbracket \boldsymbol{u} \rrbracket^{(-)}}{\partial x_{1}}, \quad x_{1}<0, \\
\left\langle\boldsymbol{\sigma}_{2}\right\rangle^{(+)}+\mathcal{A}^{(c)} \llbracket \boldsymbol{p} \rrbracket=\mathcal{B}^{(c)} \frac{\partial \llbracket \boldsymbol{u} \rrbracket^{(-)}}{\partial x_{1}}, \quad x_{1}>0,
\end{gathered}
$$

where $\mathcal{A}^{(s)}, \mathcal{B}^{(s)}: F\left(\mathbb{R}_{-}\right) \rightarrow F\left(\mathbb{R}_{-}\right)$, and $\mathcal{A}^{(c)}, \mathcal{B}^{(c)}: F\left(\mathbb{R}_{-}\right) \rightarrow F\left(\mathbb{R}_{+}\right)$are the following matrix operators

$$
\begin{gathered}
\mathcal{A}^{(s)}=\frac{b}{2\left(b^{2}-d^{2}\right)}\left[(b \alpha-d \gamma) \boldsymbol{I}+(d \alpha-b \gamma) \boldsymbol{E} \mathcal{S}^{(s)}\right], \quad \mathcal{B}^{(s)}=-\frac{1}{b^{2}-d^{2}}\left[b \boldsymbol{I} \mathcal{S}^{(s)}-d \boldsymbol{E}\right] . \\
\mathcal{A}^{(c)}=\frac{b(d \alpha-b \gamma)}{2\left(b^{2}-d^{2}\right)} \boldsymbol{E} \mathcal{S}^{(c)}, \quad \mathcal{B}^{(c)}=-\frac{b}{b^{2}-d^{2}} \boldsymbol{I S}^{(c)} .
\end{gathered}
$$

Equations (50) and (51), together with the definition of operators (52) and (53), form the system of integral identities for Mode I/II deformation. In particular, (50) is a system of two singular integral equations and this system is coupled, in general.

Note that, in the case where the Dundurs parameter $d$ vanishes, the operators can be written as

$$
\begin{gathered}
\mathcal{A}^{(s)}=\frac{\alpha}{2} \boldsymbol{I}-\frac{\gamma}{2} \boldsymbol{E} \mathcal{S}^{(s)}, \quad \mathcal{B}^{(s)}=-\frac{1}{b} \boldsymbol{I S}^{(s)} . \\
\mathcal{A}^{(c)}=-\frac{\gamma}{2} \boldsymbol{E} \mathcal{S}^{(c)}, \quad \mathcal{B}^{(c)}=-\frac{1}{b} \boldsymbol{I} \mathcal{S}^{(c)} .
\end{gathered}
$$

This means that the system (50) decouples in the case $d=0$ and reduces to

$$
-\frac{1}{b} \mathcal{S}^{(s)} \frac{\partial \llbracket \boldsymbol{u} \rrbracket^{(-)}}{\partial x_{1}}=\langle\boldsymbol{p}\rangle+\left(\frac{\alpha}{2} \boldsymbol{I}-\frac{\gamma}{2} \boldsymbol{E} \mathcal{S}^{(s)}\right) \llbracket \boldsymbol{p} \rrbracket, \quad x_{1}<0 .
$$

In the particular case of a homogeneous material, we additionally have $\alpha=0, b=2(1-\nu) / \mu$ and $\gamma=(1-2 \nu) /[2(1-\nu)]$, so that the matrix operators simplify further to the form:

$$
\mathcal{A}^{(s, c)}=-\frac{(1-2 \nu)}{4(1-\nu)} \boldsymbol{E} \mathcal{S}^{(s, c)}, \quad \mathcal{B}^{(s, c)}=-\frac{\mu}{2(1-\nu)} \boldsymbol{I S}^{(s, c)} .
$$

and the singular integral equation (56) simplifies to

$$
-\frac{\mu}{2} \mathcal{S}^{(s)} \frac{\partial \llbracket \boldsymbol{u} \rrbracket^{(-)}}{\partial x_{1}}=(1-\nu)\langle\boldsymbol{p}\rangle-\frac{1-2 \nu}{4} \boldsymbol{E} \mathcal{S}^{(s)} \llbracket \boldsymbol{p} \rrbracket, \quad x_{1}<0 .
$$

In the case of symmetrical load $(\llbracket \boldsymbol{p} \rrbracket=0)$ this formula can be found for example in Rice (1968). It differs from the Mode III case (35) only for the coefficient in front of $\langle\boldsymbol{p}\rangle$. If in addition $\nu=0$, then the two formulas fully coincide, altough one is scalar and the other is vector one.

The solution of either equation (56) or (58), allowing for the calculation of the crack opening related to a given load applied on the crack faces, requires the inversion of the operator $S^{(s)}$, which is discussed in details in Appendix A. To invert the operator (50) in the general case, one needs to use results from Vekua (1970) where the systems of singular integral equations are discussed and analysed thoroughly.

However, we propose here a simple way to decouple the system (50) by acting on it by the following operator:

$$
\boldsymbol{R}=b \boldsymbol{I} \mathcal{S}^{(s)}+d \boldsymbol{E} .
$$

As a result, equation (50) decouples as follows

$$
(1-p \mathcal{K}) \frac{\partial \llbracket \boldsymbol{u} \rrbracket^{(-)}}{\partial x_{1}}=\frac{1}{2}\left\{b \alpha \boldsymbol{I} \mathcal{S}^{(s)}+\boldsymbol{E}[b \gamma+p(d \alpha-b \gamma) \mathcal{K}]\right\} \llbracket \boldsymbol{p} \rrbracket+\left(b \boldsymbol{I} \mathcal{S}^{(s)}+d \boldsymbol{E}\right)\langle\boldsymbol{p}\rangle
$$


where we have introduced a new bimaterial constant $(0<p \leq 1)$

$$
p=\frac{b^{2}}{b^{2}-d^{2}} \text {. }
$$

In deriving (60) we used results from Muskhelishvili (1946) to prove that

$$
\left(\mathcal{S}^{(s)}\right)^{2}=-\mathcal{I}+\mathcal{K}
$$

where $\mathcal{I}$ is the identity operator and $\mathcal{K}: F\left(\mathbb{R}_{-}\right) \rightarrow F\left(\mathbb{R}_{-}\right)$is a compact operator given by:

$$
\mathcal{K} \varphi=\frac{1}{\pi^{2}} \int_{-\infty}^{0} \frac{\log \left|\xi / x_{1}\right|}{x_{1}-\xi} \varphi(\xi) d \xi
$$

It is easy to see that in the case of a homogeneous body $(d=0, \alpha=0$ and thus $p=1)$ the equation (60) is equivalent to (58). In the general case, the operator $1-p \mathcal{K}$ can be analytically inverted again using results from Muskhelishvili (1946). Alternatively, the Fredholm equation (60) can be easily inverted by numerical techniques.

\section{General 3D case}

For the case of an elastic bimaterial space, there are three linearly independent weight functions, from which we can construct the weight function tensors by ordering the three components of each weight function in columns of $3 \times 3$ matrices (Piccolroaz et al., 2007; Piccolroaz et al., 2011)

$$
\boldsymbol{U}=\left[\begin{array}{ccc}
U_{1}^{1} & U_{1}^{2} & U_{1}^{3} \\
U_{2}^{1} & U_{2}^{2} & U_{2}^{3} \\
U_{3}^{1} & U_{3}^{2} & U_{3}^{3}
\end{array}\right], \quad \boldsymbol{\Sigma}_{2}=\left[\begin{array}{ccc}
\Sigma_{21}^{1} & \Sigma_{21}^{2} & \Sigma_{21}^{3} \\
\Sigma_{22}^{1} & \Sigma_{22}^{2} & \Sigma_{22}^{3} \\
\Sigma_{23}^{1} & \Sigma_{23}^{2} & \Sigma_{23}^{3}
\end{array}\right]
$$

Assuming again perfect interface (so that $\llbracket \boldsymbol{\sigma}_{2} \rrbracket^{(+)}=\llbracket \boldsymbol{u} \rrbracket^{(+)}=0$ ), the Betti identity, relating the physical solution $\boldsymbol{u}=\left[u_{1}, u_{2}, u_{3}\right]^{T}, \boldsymbol{\sigma}_{2}=\left[\sigma_{21}, \sigma_{22},, \sigma_{23}\right]^{T}$ with the weight function $\boldsymbol{U}, \boldsymbol{\Sigma}_{2}$, writes

$$
\boldsymbol{R} \llbracket \boldsymbol{U} \rrbracket \circledast\left\langle\boldsymbol{\sigma}_{2}\right\rangle^{(+)}-\boldsymbol{R}\left\langle\boldsymbol{\Sigma}_{2}\right\rangle \circledast \llbracket \boldsymbol{u} \rrbracket^{(-)}=-\boldsymbol{R} \llbracket \boldsymbol{U} \rrbracket \circledast\langle\boldsymbol{p}\rangle-\boldsymbol{R}\langle\boldsymbol{U}\rangle \circledast \llbracket \boldsymbol{p} \rrbracket,
$$

where the symbol $\circledast$ denotes convolution with respect to both variables $x_{1}$ and $x_{3}$ and $\boldsymbol{R}$ is the matrix defined in Sec. 1

Let us introduce the Fourier transform with respect to the $x_{1}$ and $x_{3}$ coordinates as follows

$$
\bar{f}\left(\beta, x_{2}, \lambda\right)=\int_{-\infty}^{\infty} \int_{-\infty}^{\infty} f\left(x_{1}, x_{2}, x_{3}\right) e^{i \beta x_{1}+i \lambda x_{3}} d x_{1} d x_{3} .
$$

Taking the Fourier transform of (65) we obtain

$$
\llbracket \overline{\boldsymbol{U}} \rrbracket^{T} \boldsymbol{R}\left\langle\overline{\boldsymbol{\sigma}}_{2}\right\rangle^{+}-\left\langle\overline{\boldsymbol{\Sigma}}_{2}\right\rangle^{T} \boldsymbol{R} \llbracket \overline{\boldsymbol{u}} \rrbracket^{-}=-\llbracket \overline{\boldsymbol{U}} \rrbracket^{T} \boldsymbol{R}\langle\overline{\boldsymbol{p}}\rangle-\langle\overline{\boldsymbol{U}}\rangle^{T} \boldsymbol{R} \llbracket \overline{\boldsymbol{p}} \rrbracket .
$$

Multiplying both sides of (67) by $\boldsymbol{R}^{-1} \llbracket \overline{\boldsymbol{U}} \rrbracket^{-T}$ we get

$$
\langle\overline{\boldsymbol{p}}\rangle^{-}+\boldsymbol{A} \llbracket \overline{\boldsymbol{p}} \rrbracket^{-}=-\left\langle\overline{\boldsymbol{\sigma}}_{2}\right\rangle^{+}+\boldsymbol{B} \llbracket \overline{\boldsymbol{u}} \rrbracket^{-} .
$$

where

$$
\boldsymbol{A}=\boldsymbol{R}^{-1} \llbracket \overline{\boldsymbol{U}} \rrbracket^{-T}\langle\overline{\boldsymbol{U}}\rangle^{T} \boldsymbol{R}, \quad \boldsymbol{B}=\boldsymbol{R}^{-1} \llbracket \overline{\boldsymbol{U}} \rrbracket^{-T}\left\langle\overline{\boldsymbol{\Sigma}}_{2}\right\rangle^{T} \boldsymbol{R} .
$$

The two matrices $\boldsymbol{A}$ and $\boldsymbol{B}$ can be obtained from the following relationships between symmetric and skew-symmetric weight functions (Antipov, 1999; Piccolroaz et al., 2011)

$$
\llbracket \overline{\boldsymbol{U}} \rrbracket=\frac{1}{\rho} \boldsymbol{G}\left\langle\overline{\boldsymbol{\Sigma}}_{2}\right\rangle, \quad\langle\overline{\boldsymbol{U}}\rangle=\frac{1}{\rho} \boldsymbol{F}\left\langle\overline{\boldsymbol{\Sigma}}_{2}\right\rangle,
$$


where $\rho=\sqrt{\beta^{2}+\lambda^{2}}$,

$$
\begin{gathered}
\boldsymbol{G}(\beta, \lambda)=-\frac{1}{\rho^{2}}\left(b \rho^{2} \boldsymbol{I}+e \boldsymbol{E}_{2}+i d \rho \boldsymbol{E}_{3}\right), \quad \boldsymbol{F}(\beta, \lambda)=-\frac{1}{2 \rho^{2}}\left(b \alpha \rho^{2} \boldsymbol{I}+f \boldsymbol{E}_{2}+i b \gamma \rho \boldsymbol{E}_{3}\right) . \\
\boldsymbol{E}_{2}(\beta, \lambda)=\left[\begin{array}{ccc}
\lambda^{2} & 0 & -\beta \lambda \\
0 & 0 & 0 \\
-\beta \lambda & 0 & \beta^{2}
\end{array}\right], \quad \boldsymbol{E}_{3}(\beta, \lambda)=\left[\begin{array}{ccc}
0 & -\beta & 0 \\
\beta & 0 & \lambda \\
0 & -\lambda & 0
\end{array}\right],
\end{gathered}
$$

$\boldsymbol{I}$ is the $3 \times 3$ identity matrix, $\boldsymbol{E}_{2} \boldsymbol{E}_{3}=\underline{0}$ and $e, f$ are the following bimaterial parameters:

$$
e=\frac{\nu_{+}}{\mu_{+}}+\frac{\nu_{-}}{\mu_{-}}, \quad f=\frac{\nu_{+}}{\mu_{+}}-\frac{\nu_{-}}{\mu_{-}} .
$$

As a result, we obtain

$$
\begin{gathered}
\boldsymbol{A}(\beta, \lambda)=\frac{1}{2\left(b^{2}-d^{2}\right)(b+e) \rho^{2}} \times \\
\left\{b(b \alpha-d \gamma)(b+e) \rho^{2} \boldsymbol{I}+\left[b d \gamma(b+e)-b \alpha\left(b e+d^{2}\right)+\left(b^{2}-d^{2}\right) f\right] \boldsymbol{E}_{2}+i b(b \gamma-d \alpha)(b+e) \rho \boldsymbol{E}_{3}\right\}, \\
\boldsymbol{B}(\beta, \lambda)=\frac{1}{\left(b^{2}-d^{2}\right)(b+e) \rho}\left\{-b(b+e) \rho^{2} \boldsymbol{I}+\left(b e+d^{2}\right) \boldsymbol{E}_{2}+i d(b+e) \rho \boldsymbol{E}_{3}\right\} .
\end{gathered}
$$

By inversion of the Fourier transform for the two cases $x_{1}<0$ and $x_{1}>0$, we obtain the two formulae

$$
\begin{gathered}
\langle\boldsymbol{p}\rangle+\mathcal{F}_{x_{1}<0}^{-1} \mathcal{F}_{x_{3}}^{-1}[\boldsymbol{A} \llbracket \overline{\boldsymbol{p}} \rrbracket]=\mathcal{F}_{x_{1}<0}^{-1} \mathcal{F}_{x_{3}}^{-1}\left[\boldsymbol{B} \llbracket \overline{\boldsymbol{u}} \rrbracket^{-}\right], \\
\left\langle\boldsymbol{\sigma}_{2}\right\rangle^{(+)}+\mathcal{F}_{x_{1}>0}^{-1} \mathcal{F}_{x_{3}}^{-1}[\boldsymbol{A} \llbracket \overline{\boldsymbol{p}} \rrbracket]=\mathcal{F}_{x_{1}>0}^{-1} \mathcal{F}_{x_{3}}^{-1}\left[\boldsymbol{B} \llbracket \overline{\boldsymbol{u}} \rrbracket^{-}\right] .
\end{gathered}
$$

We need now to perform the double Fourier inversion. The details are given in Appendix E The final result is given in terms of the following operators

$$
\begin{gathered}
\mathcal{Q} \varphi=\frac{1}{\pi \sqrt{x_{1}^{2}+x_{3}^{2}}} \circledast \varphi\left(x_{1}, x_{3}\right)=\frac{1}{\pi} \int_{-\infty}^{\infty} \int_{-\infty}^{\infty} \frac{\varphi\left(\xi_{1}, \xi_{3}\right)}{\sqrt{\left(x_{1}-\xi_{1}\right)^{2}+\left(x_{3}-\xi_{3}\right)^{2}}} d \xi_{1} d \xi_{3}, \\
\mathcal{Q}_{j} \varphi=\frac{x_{j}}{\pi\left(x_{1}^{2}+x_{3}^{2}\right)} \circledast \varphi\left(x_{1}, x_{3}\right)=\frac{1}{\pi} \int_{-\infty}^{\infty} \int_{-\infty}^{\infty} \frac{\left(x_{j}-\xi_{j}\right) \varphi\left(\xi_{1}, \xi_{3}\right)}{\left(x_{1}-\xi_{1}\right)^{2}+\left(x_{3}-\xi_{3}\right)^{2}} d \xi_{1} d \xi_{3}, \quad j=1,3 . \\
\mathcal{Q}^{(s, c)}=\mathcal{P}_{(-,+)} \mathcal{Q P}_{-}, \quad \mathcal{Q}_{j}^{(s, c)}=\mathcal{P}_{(-,+)} \mathcal{Q}_{j} \mathcal{P}_{-}, \quad j=1,3 .
\end{gathered}
$$

The integral identities for $3 \mathrm{D}$ elasticity are given by

$$
\begin{gathered}
\langle\boldsymbol{p}\rangle+\mathcal{A}^{(s)} \llbracket \boldsymbol{p} \rrbracket=\mathcal{B}^{(s)} \llbracket \boldsymbol{u} \rrbracket^{(-)}, \quad x_{1}<0, \\
\left\langle\boldsymbol{\sigma}_{2}\right\rangle^{(+)}+\mathcal{A}^{(c)} \llbracket \boldsymbol{p} \rrbracket=\mathcal{B}^{(c)} \llbracket \boldsymbol{u} \rrbracket^{(-)}, \quad x_{1}>0,
\end{gathered}
$$

where

$$
\begin{aligned}
& \mathcal{A}^{(s)}=\frac{1}{2\left(b^{2}-d^{2}\right)(b+e)} \times \\
& \left\{b(b \alpha-d \gamma)(b+e) \boldsymbol{I}+\frac{1}{2}\left[b d \gamma(b+e)-b \alpha\left(b e+d^{2}\right)+\left(b^{2}-d^{2}\right) f\right] \mathcal{E}_{2}^{(s)}\right. \\
& \left.-\frac{1}{2} b(b \gamma-d \alpha)(b+e) \mathcal{Q}^{(s)} \boldsymbol{E}_{3}\left(\frac{\partial}{\partial x_{1}}, \frac{\partial}{\partial x_{3}}\right)\right\},
\end{aligned}
$$




$$
\begin{aligned}
& \mathcal{B}^{(s)}=\frac{1}{2\left(b^{2}-d^{2}\right)(b+e)} \times \\
& \left\{b(b+e) \boldsymbol{I} \mathcal{Q}^{(s)}\left(\frac{\partial^{2}}{\partial x_{1}^{2}}+\frac{\partial^{2}}{\partial x_{3}^{2}}\right)-\left(b e+d^{2}\right) \mathcal{Q}^{(s)} \boldsymbol{E}_{2}\left(\frac{\partial}{\partial x_{1}}, \frac{\partial}{\partial x_{3}}\right)-2 d(b+e) \boldsymbol{E}_{3}\left(\frac{\partial}{\partial x_{1}}, \frac{\partial}{\partial x_{3}}\right)\right\}, \\
& \mathcal{A}^{(c)}=\frac{1}{2\left(b^{2}-d^{2}\right)(b+e)} \times \\
& \left\{\frac{1}{2}\left[b d \gamma(b+e)-b \alpha\left(b e+d^{2}\right)+\left(b^{2}-d^{2}\right) f\right] \mathcal{E}_{2}^{(c)}-\frac{1}{2} b(b \gamma-d \alpha)(b+e) \mathcal{Q}^{(c)} \boldsymbol{E}_{3}\left(\frac{\partial}{\partial x_{1}}, \frac{\partial}{\partial x_{3}}\right)\right\} \\
& \mathcal{B}^{(c)}=\frac{1}{2\left(b^{2}-d^{2}\right)(b+e)}\left\{b(b+e) \boldsymbol{I} \mathcal{Q}^{(c)}\left(\frac{\partial^{2}}{\partial x_{1}^{2}}+\frac{\partial^{2}}{\partial x_{3}^{2}}\right)-\left(b e+d^{2}\right) \mathcal{Q}^{(c)} \boldsymbol{E}_{2}\left(\frac{\partial}{\partial x_{1}}, \frac{\partial}{\partial x_{3}}\right)\right\}
\end{aligned}
$$

and the matrix operator $\mathcal{E}_{2}^{(s, c)}$ is defined as

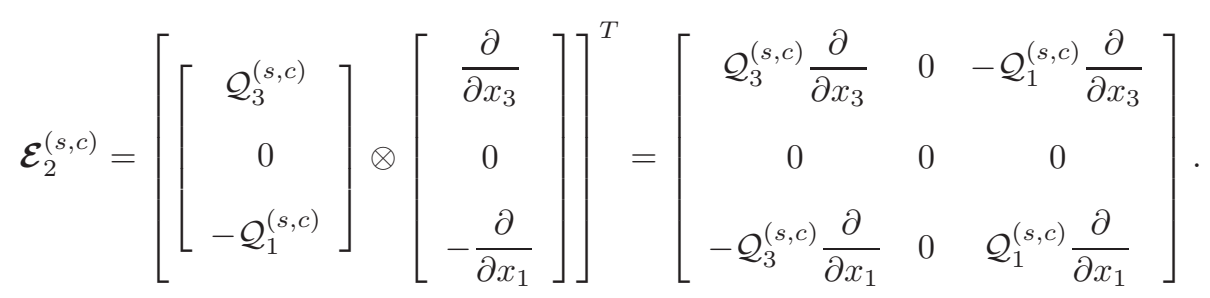

In the case of homogeneous body the matrix operators $\mathcal{A}^{(s)}, \mathcal{B}^{(s)}, \mathcal{A}^{(c)}, \mathcal{B}^{(c)}$ simplify to

$$
\mathcal{A}^{(s, c)}=-\frac{1-2 \nu}{8(1-\nu)} \mathcal{Q}^{(s, c)}\left[\begin{array}{ccc}
0 & -\frac{\partial}{\partial x_{1}} & 0 \\
\frac{\partial}{\partial x_{1}} & 0 & \frac{\partial}{\partial x_{3}} \\
0 & -\frac{\partial}{\partial x_{3}} & 0
\end{array}\right],
$$

We note that the singular integral formulation (81) for the case of a homogeneous body and symmetrical forces $(\llbracket \boldsymbol{p} \rrbracket=0)$ is equivalent to the formulation given by Weaver (1977) and Budiansky and Rice (1979). To show this it is sufficient to use integration by parts, taking into account the behaviour of the displacements jump at infinity.

\section{Conclusions}

The singular integral formulation for a three-dimensional semi-infinite interfacial crack has been derived in explicit form by means of fundamental properties of linear elasticity and integral transforms. This formulation for interfacial cracks seems to be unknown in the literature. The method of derivation avoids the use of Green's function and the tedious limiting process involved in the general procedure. The identities are important in applications especially in case of multiphysics problems where the elasticity equations are coupled with other concurrent phenomena, such as in hydraulic fracturing. 
Acknowledgement. A.P. and G.M. gratefully acknowledge the support from the European Union Seventh Framework Programme under contract numbers PIEF-GA-2009-252857 and PIAP-GA-2009251475 , respectively.

\section{References}

[1] Antipov, Y.A., An exact solution of the 3-D-problem of an interface semi-infinite plane crack. $J$. Mech. Phys. Solids 47, 1051-1093, 1999.

[2] Barber, J.R., Elasticity. Kluwer Academic Publishers, Dordrecht, 2002.

[3] Bercial-Velez, J.P., Antipov, Y.A., Movchan, A.B., High-order asymptotics and perturbation problems for 3D interfacial cracks. J. Mech. Phys. Solids 53, 1128-1162, 2005.

[4] Bigoni, D., Capuani, D., Green's function for incremental nonlinear elasticity: shear bands and boundary integral formulation. J. Mech. Phys. Solids 50, 471-500, 2002.

[5] Bigoni, D., Dal Corso, F., The unrestrainable growth of a shear band in a prestressed material. Proc. R. Soc. A 464, 2365-2390, 2008.

[6] Budiansky, B., Rice, J.R., An integral equation for dynamic elastic response of an isolated 3-D crack. Wave motion 1, 187-192, 1979.

[7] Bueckner, H. F., A novel principle for the computation of stress intensity factors. Z. Angew. Math. Mech. 50, 529-546, 1970.

[8] Gakhov, F.D., Cherski, Y.I., Equations of the convolution type. Nauka, Moscow, 1978.

[9] Garagash, D., Detournay,E., The tip region of a fluid-driven fracture in elastic medium. ASME J. Appl. Mech. 67, 183-192, 2000.

[10] Gohberg, I., Krein, M.G., Systems of integral equations on a half line with kernels depending on the difference of arguments. Uspekhi Mat. Nauk. 13, 3-72, 1958.

[11] Gradshteyn, I.S., Ryzhik, I.M., Table of integrals, series, and products. Academic Press, New York, 1965.

[12] Krein, M.G., Integral equations on a half-line with kernel depending upon the difference of the arguments. Uspekhi Mat. Nauk. 13, 3-120, 1958.

[13] Kupradze, V., Gegelia, T., Basheleishvili, M., Burchuladze, T., Three-dimensional problems of the mathematical theory of elasticity and thermoelasticity. North-Holland, Amsterdam, 1979.

[14] Linkov, A.M., Zubkov, V.V., Kheib, M.A., A method of solving three-dimensional problems of seam workings and geological faults. J. Min. Sci. 33, 295-315, 1997.

[15] Mikhlin, S., Prössdorf, S., Singular integral operators. Akademie-Verlag, Berlin, 1980.

[16] Muskhelishvili, N.I., Singular integral equations. Moscow, 1946.

[17] Muskhelishvili, N.I., Some basic problems of the mathematical theory of elasticity. Noordhoff, 1953.

[18] Piccolroaz, A., Mishuris, G., Movchan, A.B., Evaluation of the Lazarus-Leblond constants in the asymptotic model of the interfacial wavy crack. J. Mech. Phys. Solids 55, 1575-1600, 2007.

[19] Piccolroaz, A., Mishuris, G., Movchan, A.B., Symmetric and skew-symmetric weight functions in 2D perturbation models for semi-infinite interfacial cracks, J. Mech. Phys. Solids 57, 1657-1682, 2009.

[20] Piccolroaz, A., Mishuris, G., Movchan, A.B., 3D skew-symmetric weight functions for semi-infinite interfacial cracks. To appear. 
[21] Prössdorf, S., Some classes of singular equations. Akademie-Verlag, Berlin, 1974.

[22] Rice, J.R., Mathematical analysis in the mechanics of fracture, in Fracture: An advanced treatise, Vol II, Academic Press, San Diego, 191-311, 1968.

[23] Vekua, N.P., Systems of singular integral equations. Nauka, Moscow, 1970.

[24] Weaver, J., Three-dimensional crack analysis. Int. J. Solids Struct. 13, 321-330, 1977.

[25] Willis, J.R., Movchan, A.B., Dynamic weight functions for a moving crack. I. Mode I loading. J. Mech. Phys. Solids 43, 319-341, 1995.

\section{A Inversion of the 2D singular operator $S^{(s)}$}

The inversion formula for the singular integral equation

$$
\psi\left(x_{1}\right)=S^{(s)} \varphi\left(x_{1}\right)=\frac{1}{\pi} \int_{-\infty}^{0} \frac{\varphi(\xi)}{x_{1}-\xi} d \xi, \quad x_{1}<0,
$$

strongly depends on the properties of the known function $\psi\left(x_{1}\right)$. Indeed, let us first assume that the function $\psi\left(x_{1}\right)$ has compact support $-a \leq x_{1} \leq-b$, where $a$ and $b$ are positive constants, and belongs to a Hölder class, as it usually takes place in the classical 2D elasticity (Muskhelishvili, 1946). Then the inversion formula can be found in Muskhelishvili (1946) (see also Rice, 1968)

$$
\varphi\left(x_{1}\right)=\left(S^{(s)}\right)_{1}^{-1} \psi\left(x_{1}\right)=-\frac{1}{\pi} \int_{-\infty}^{0} \sqrt{\frac{\xi}{x_{1}}} \frac{\psi(\xi)}{x_{1}-\xi} d \xi, \quad x_{1}<0 .
$$

Note that under such assumptions

$$
\varphi\left(x_{1}\right) \sim \frac{K_{0}}{\sqrt{-x_{1}}}, \quad x_{1} \rightarrow 0^{-}, \quad \varphi\left(x_{1}\right) \sim \frac{K_{\infty}}{\left(-x_{1}\right)^{3 / 2}}, \quad x_{1} \rightarrow-\infty .
$$

Here the constants $K_{0}$ and $K_{\infty}$ ( $K_{0}$ is the so-called stress intensity factor) are determined by the formulae

$$
K_{0}=-\frac{1}{\pi} \int_{-\infty}^{0} \frac{\psi(\xi)}{\sqrt{-\xi}} d \xi, \quad K_{\infty}=\frac{1}{\pi} \int_{-\infty}^{0} \psi(\xi) \sqrt{-\xi} d \xi
$$

Let us now consider the case where the function $\psi\left(x_{1}\right)$ extends over the whole negative semi-axis, having the following behaviour at zero and infinity:

$$
\psi\left(x_{1}\right) \sim \frac{\psi_{0}}{\left(-x_{1}\right)^{\alpha_{0}}}, \quad x_{1} \rightarrow 0^{-}, \quad \psi\left(x_{1}\right) \sim \frac{\psi_{\infty}}{\left(-x_{1}\right)^{\alpha_{\infty}}}, \quad x_{1} \rightarrow-\infty .
$$

If we assume that $\alpha_{0}<1 / 2$ and $\alpha_{\infty}>3 / 2$ then the inversion formula (A.2) is still valid and leads to the asymptotics (A.3). In the case $\alpha_{0}<1 / 2$ and $1 / 2<\alpha_{\infty}<3 / 2$ the inversion formula (A.2) remains true, the behaviour of the function $\psi\left(x_{1}\right)$ near zero is the same as in $(\mathrm{A.3})_{1}$, while the behaviour at infinity ${ }_{2}$. 2 changes to:

$$
\varphi\left(x_{1}\right) \sim \frac{-\pi \psi_{\infty} \sin \left(\pi \alpha_{\infty}\right)}{\left(-x_{1}\right)^{\alpha_{\infty}}}, \quad x_{1} \rightarrow-\infty .
$$

However, there are situations (such as some problems arising in hydraulic fracturing, see Garagash and Detournay, 2000) where the behaviour of the function $\psi\left(x_{1}\right)$ at infinity is worst, so that $\alpha_{\infty}$ in (A.5) is in the range $0<\alpha_{\infty}<1 / 2$. In such cases, the classic inversion formula (A.2) is not any longer applicable and another inversion formula should be used instead. In Garagash and Detournay (2000) the formula

$$
\varphi\left(x_{1}\right)=\left(S^{(s)}\right)_{2}^{-1} \psi\left(x_{1}\right)=-\frac{C_{0}}{\left(-x_{1}\right)^{1 / 2}}-\frac{1}{\pi} \int_{-\infty}^{0} \sqrt{\frac{x_{1}}{\xi}} \frac{\psi(\xi)}{x_{1}-\xi} d \xi, \quad x_{1}<0,
$$


was derived by easy and elegant arguments leaving, however, the constant $C_{0}$ unknown.

Here we present an inversion formula accurately derived under slightly more restricted conditions on the behaviour of the function $\psi\left(x_{1}\right)$ at infinity. Namely we assume that

$$
\psi\left(x_{1}\right)=\psi_{\infty}\left(x_{1}\right)+O\left[\frac{1}{\left(-x_{1}\right)^{\beta}}\right], \quad x_{1} \rightarrow-\infty,
$$

where

$$
\psi_{\infty}\left(x_{1}\right)=\sum_{j=1}^{N} \frac{\psi_{\infty}^{(j)}}{\left(-x_{1}\right)^{\alpha_{\infty}^{(j)}}}
$$

and $0<\alpha_{\infty}^{(j)}<1 / 2$ and $\beta>1 / 2$. The alternative formula reads

$$
\varphi\left(x_{1}\right)=\left(S^{(s)}\right)_{2}^{-1} \psi\left(x_{1}\right)=\sum_{j=1}^{N} \tan \left(\pi \alpha_{\infty}^{(j)}\right) \frac{\psi_{\infty}^{(j)}}{\left(-x_{1}\right)^{\alpha_{\infty}^{(j)}}}-\frac{1}{\pi} \int_{-\infty}^{0} \sqrt{\frac{\xi}{x_{1}}} \frac{\psi(\xi)-\psi_{\infty}(\xi)}{x_{1}-\xi} d \xi, \quad x_{1}<0 .
$$

Note that this formula can be rewritten in the equivalent form (A.7), where the constant $C_{0}$ can be determined by

$$
C_{0}=\frac{1}{\pi} \int_{-\infty}^{0} \frac{\psi(\xi)-\psi_{\infty}(\xi)}{(-\xi)^{1 / 2}} d \xi
$$

\section{B Reduction of the identities from 3D to 2D case}

The integral identities for 3D elasticity can be reduced to the 2D case by assuming that all the mechanical fields are independent of the $x_{3}$ coordinate. We start by showing that if the fields $\boldsymbol{u}, \boldsymbol{\sigma}_{2}, \boldsymbol{p}$ are independent of $x_{3}$ then the convolution with respect to $x_{1}$ and $x_{3}$, denoted by $\circledast$, reduces to the convolution with respect to the $x_{1}$ coordinate only, denoted by $*$.

In particular, we have the following formulae (proved in Appendix D TheoremD.1). If $\varphi$ is a function of $x_{1}$ only, then

$$
\begin{array}{cc}
\frac{1}{\sqrt{x_{1}^{2}+x_{3}^{2}}} \circledast \frac{\partial^{2} \varphi}{\partial x_{1}^{2}}=-2 \frac{1}{x_{1}} * \frac{\partial \varphi}{\partial x_{1}} & \rightsquigarrow \mathcal{Q} \frac{\partial^{2} \varphi}{\partial x_{1}^{2}}=-2 \mathcal{S} \frac{\partial \varphi}{\partial x_{1}} \\
\frac{1}{\sqrt{x_{1}^{2}+x_{3}^{2}}} \circledast \frac{\partial \varphi}{\partial x_{1}}=-2 \frac{1}{x_{1}} * \varphi \quad & \rightsquigarrow \quad \mathcal{Q} \frac{\partial \varphi}{\partial x_{1}}=-2 \mathcal{S} \varphi .
\end{array}
$$

We also make use of the following identities (proved in Appendix D, Theorems D.2 and D.3):

$$
\begin{gathered}
\frac{x_{1}}{x_{1}^{2}+x_{3}^{2}} \circledast \frac{\partial \varphi}{\partial x_{1}}+\frac{x_{3}}{x_{1}^{2}+x_{3}^{2}} \circledast \frac{\partial \varphi}{\partial x_{3}}=2 \pi \varphi \quad \rightsquigarrow \quad \mathcal{Q}_{1} \frac{\partial \varphi}{\partial x_{1}}+\mathcal{Q}_{3} \frac{\partial \varphi}{\partial x_{3}}=2 \varphi, \\
\frac{x_{3}}{x_{1}^{2}+x_{3}^{2}} \circledast \frac{\partial \varphi}{\partial x_{1}}=\frac{x_{1}}{x_{1}^{2}+x_{3}^{2}} \circledast \frac{\partial \varphi}{\partial x_{3}}
\end{gathered}
$$

If $\varphi$ is a function of $x_{1}$ only then the identities (B.3) and (B.4) yield

$$
\begin{array}{ccc}
\frac{x_{1}}{x_{1}^{2}+x_{3}^{2}} \circledast \frac{\partial \varphi}{\partial x_{1}}=2 \pi \varphi & \rightsquigarrow \quad \mathcal{Q}_{1} \frac{\partial \varphi}{\partial x_{1}}=2 \varphi, \\
\frac{x_{3}}{x_{1}^{2}+x_{3}^{2}} \circledast \frac{\partial \varphi}{\partial x_{1}}=0 \quad \rightsquigarrow \quad \mathcal{Q}_{3} \frac{\partial \varphi}{\partial x_{1}}=0 .
\end{array}
$$

Using the formulae (B.1), (B.2), (B.5) and (B.6), the integral identity (81) for 3D elasticity reduces to

$$
\langle\boldsymbol{p}\rangle+\frac{b}{2\left(b^{2}-d^{2}\right)}\left[\begin{array}{ccc}
(b \alpha-d \gamma) & (d \alpha-b \gamma) \mathcal{S}^{(s)} & 0 \\
-(d \alpha-b \gamma) \mathcal{S}^{(s)} & (b \alpha-d \gamma) & 0 \\
0 & 0 & 0
\end{array}\right] \llbracket \boldsymbol{p} \rrbracket+\frac{\eta}{2}\left[\begin{array}{ccc}
0 & 0 & 0 \\
0 & 0 & 0 \\
0 & 0 & 1
\end{array}\right] \llbracket \boldsymbol{p} \rrbracket=
$$




$$
-\frac{1}{b^{2}-d^{2}}\left[\begin{array}{ccc}
b \mathcal{S}^{(s)} \frac{\partial}{\partial x_{1}} & -d \frac{\partial}{\partial x_{1}} & 0 \\
d \frac{\partial}{\partial x_{1}} & b \mathcal{S}^{(s)} \frac{\partial}{\partial x_{1}} & 0 \\
0 & 0 & 0
\end{array}\right] \llbracket \boldsymbol{u} \rrbracket^{(-)}-\frac{1}{b+e}\left[\begin{array}{ccc}
0 & 0 & 0 \\
0 & 0 & 0 \\
0 & 0 & \mathcal{S}^{(s)} \frac{\partial}{\partial x_{1}}
\end{array}\right] \llbracket \boldsymbol{u} \rrbracket^{(-)} .
$$

This formula is fully consistent with the formulation for 2 D elasticity given in Secs. 3 and 4 . In a similar way, also the formula (82) can be shown to reduce to the 2D formulation.

\section{$\mathrm{C}$ Illustrative example in $2 \mathrm{D}$ case: point forces applied at the crack faces}

In this section, we illustrate the use of the singular integral identities in the 2D case, when the loading is given in terms of point forces applied at the crack faces. We analyse first the antiplane problem and then the vector problem.

Mode III. Symmetrical point forces. Assume that the loading is given as two symmetrical point forces applied on the crack faces, at a distance $a$ from the crack tip, and directed along the $x_{3}$-axis,

$$
\left\langle p_{3}\right\rangle\left(x_{1}\right)=-F \delta\left(x_{1}+a\right), \quad \llbracket p_{3} \rrbracket\left(x_{1}\right)=0,
$$

where $\delta$ is the Dirac delta function.

The singular integral equation relating the applied loading with the resulting crack opening is given by (31) and, by means of the inversion formula (A.2) we obtain

$$
\frac{\partial \llbracket u_{3} \rrbracket^{(-)}}{\partial x_{1}}=-\frac{b+e}{\pi} F \int_{-\infty}^{0} \sqrt{\frac{\xi}{x_{1}}} \frac{\delta(\xi+a)}{x_{1}-\xi} d \xi=-\frac{b+e}{\pi} F \sqrt{-\frac{a}{x_{1}}} \frac{1}{x_{1}+a} .
$$

By integration of (C.2) and using the conditions that the crack opening vanishes at zero and at infinity, we get

$$
\llbracket u_{3} \rrbracket\left(x_{1}\right)= \begin{cases}\frac{2 F}{\pi}(b+e) \operatorname{arctanh} \sqrt{-\frac{x_{1}}{a}}, & -a<x_{1}<0, \\ \frac{2 F}{\pi}(b+e) \operatorname{arctanh} \sqrt{-\frac{a}{x_{1}}}, & x_{1}<-a .\end{cases}
$$

From (32) we can now obtain the corresponding tractions ahead of the crack tip, namely

$$
\left\langle\sigma_{23}\right\rangle^{(+)}\left(x_{1}\right)=-\frac{1}{\pi(b+e)} \int_{-\infty}^{0} \frac{1}{x_{1}-\xi} \frac{\partial \llbracket u_{3} \rrbracket^{(-)}}{\partial \xi} d \xi=\frac{F}{\pi} \sqrt{\frac{a}{x_{1}}} \frac{1}{x_{1}+a} .
$$

Note that the stress intensity factor in this case is

$$
K_{\mathrm{III}}=\lim _{x_{1} \rightarrow 0} \sqrt{2 \pi x_{1}}\left\langle\sigma_{23}\right\rangle^{(+)}\left(x_{1}\right)=\sqrt{\frac{2}{\pi a}} F .
$$

Mode III. Skew-symmetrical point forces. We assume now that the loading is given by two skew-symmetrical point forces again applied at a distance $a$ behind crack tip and directed along the $x_{3}$-axis,

$$
\left\langle p_{3}\right\rangle\left(x_{1}\right)=0, \quad \llbracket p_{3} \rrbracket\left(x_{1}\right)=-2 F \delta\left(x_{1}+a\right) .
$$

It is easy to show that all the formulae derived above still apply, however, they contain now the factor $\eta$, so that

$$
\frac{\partial \llbracket u_{3} \rrbracket^{(-)}}{\partial x_{1}}=-\eta \frac{b+e}{\pi} F \sqrt{-\frac{a}{x_{1}}} \frac{1}{x_{1}+a},
$$




$$
\begin{gathered}
\llbracket u_{3} \rrbracket\left(x_{1}\right)= \begin{cases}\eta \frac{2 F}{\pi}(b+e) \operatorname{arctanh} \sqrt{-\frac{x_{1}}{a}}, & -a<x_{1}<0, \\
\eta \frac{2 F}{\pi}(b+e) \operatorname{arctanh} \sqrt{-\frac{a}{x_{1}}}, & x_{1}<-a,\end{cases} \\
\left\langle\sigma_{23}\right\rangle^{(+)}\left(x_{1}\right)=\eta \frac{F}{\pi} \sqrt{\frac{a}{x_{1}}} \frac{1}{x_{1}+a},
\end{gathered}
$$

and the stress intensity factor is given by

$$
K_{\mathrm{III}}=\eta \sqrt{\frac{2}{\pi a}} F .
$$

Mode I and II. Symmetrical point forces. We consider now the vector 2D case and suppose that the loading is given as symmetrical point forces in both $x_{1}$ and $x_{2}$ directions, namely

$$
\begin{array}{ll}
\left\langle p_{1}\right\rangle\left(x_{1}\right)=-F_{1} \delta\left(x_{1}+a\right), & \llbracket p_{1} \rrbracket\left(x_{1}\right)=0, \\
\left\langle p_{2}\right\rangle\left(x_{1}\right)=-F_{2} \delta\left(x_{1}+a\right), & \llbracket p_{2} \rrbracket\left(x_{1}\right)=0 .
\end{array}
$$

For simplicity we assume that the Dundurs parameter $d$ is equal to zero, so that the system of singular equations (50) decouples

$$
-\frac{1}{b} \mathcal{S}^{(s)} \frac{\partial \llbracket u_{j} \rrbracket^{(-)}}{\partial x_{1}}=\left\langle p_{j}\right\rangle\left(x_{1}\right), \quad j=1,2 .
$$

and it is possible to obtain results in closed form. Indeed, the inversion formula (A.2) leads to

$$
\frac{\partial \llbracket u_{j} \rrbracket^{(-)}}{\partial x_{1}}=-\frac{b F_{j}}{\pi} \sqrt{-\frac{a}{x_{1}}} \frac{1}{x_{1}+a},
$$

which, after integration, gives

$$
\llbracket u_{j} \rrbracket\left(x_{1}\right)= \begin{cases}\frac{2 b F_{j}}{\pi} \operatorname{arctanh} \sqrt{-\frac{x_{1}}{a}}, & -a<x_{1}<0, \\ \frac{2 b F_{j}}{\pi} \operatorname{arctanh} \sqrt{-\frac{a}{x_{1}}}, & x_{1}<-a .\end{cases}
$$

Finally, the tractions ahead of the crack tip are calculated from (51)

$$
\left\langle\sigma_{2 j}\right\rangle^{(+)}\left(x_{1}\right)=\frac{F_{j}}{\pi} \sqrt{\frac{a}{x_{1}}} \frac{1}{x_{1}+a} .
$$

The stress intensity factors are then obtained as

$$
K_{\mathrm{I}}=\sqrt{\frac{2}{\pi a}} F_{2}, \quad K_{\mathrm{II}}=\sqrt{\frac{2}{\pi a}} F_{1} .
$$

Mode I and II. Skew-symmetrical point forces. In this case the point forces, applied at a distance $a$ behind the crack tip, have the same direction

$$
\begin{array}{ll}
\left\langle p_{1}\right\rangle\left(x_{1}\right)=0, & \llbracket p_{1} \rrbracket\left(x_{1}\right)=-2 F_{1} \delta\left(x_{1}+a\right), \\
\left\langle p_{2}\right\rangle\left(x_{1}\right)=0, & \llbracket p_{2} \rrbracket\left(x_{1}\right)=-2 F_{2} \delta\left(x_{1}+a\right) .
\end{array}
$$

Assuming again $d=0$, the solution of the system of singular integral equations (50) leads to

$$
\frac{\partial \llbracket u_{1} \rrbracket^{(-)}}{\partial x_{1}}=-\frac{b \alpha F_{1}}{\pi} \sqrt{-\frac{a}{x_{1}}} \frac{1}{x_{1}+a}-b \gamma F_{2} \delta\left(x_{1}+a\right),
$$




$$
\frac{\partial \llbracket u_{2} \rrbracket^{(-)}}{\partial x_{1}}=-\frac{b \alpha F_{2}}{\pi} \sqrt{-\frac{a}{x_{1}}} \frac{1}{x_{1}+a}+b \gamma F_{1} \delta\left(x_{1}+a\right),
$$

which after integration gives

$$
\begin{aligned}
& \llbracket u_{1} \rrbracket\left(x_{1}\right)= \begin{cases}\frac{2 b \alpha F_{1}}{\pi} \operatorname{arctanh} \sqrt{-\frac{x_{1}}{a}}, & -a<x_{1}<0, \\
\frac{2 b \alpha F_{1}}{\pi} \operatorname{arctanh} \sqrt{-\frac{a}{x_{1}}}+b \gamma F_{2}, & x_{1}<-a,\end{cases} \\
& \llbracket u_{2} \rrbracket\left(x_{1}\right)= \begin{cases}\frac{2 b \alpha F_{2}}{\pi} \operatorname{arctanh} \sqrt{-\frac{x_{1}}{a}}, & -a<x_{1}<0, \\
\frac{2 b \alpha F_{2}}{\pi} \operatorname{arctanh} \sqrt{-\frac{a}{x_{1}}}-b \gamma F_{1}, & x_{1}<-a .\end{cases}
\end{aligned}
$$

The tractions ahead of the crack tip are obtained from (51) and read

$$
\begin{aligned}
& \left\langle\sigma_{21}\right\rangle^{(+)}\left(x_{1}\right)=-\frac{1}{b} \mathcal{S}^{(c)} \frac{\partial \llbracket u_{1} \rrbracket^{(-)}}{\partial x_{1}}+\frac{\gamma}{2} \mathcal{S}^{(c)} \llbracket p_{2} \rrbracket\left(x_{1}\right)=\alpha \frac{F_{1}}{\pi} \sqrt{\frac{a}{x_{1}}} \frac{1}{x_{1}+a}, \\
& \left\langle\sigma_{22}\right\rangle^{(+)}\left(x_{1}\right)=-\frac{1}{b} \mathcal{S}^{(c)} \frac{\partial \llbracket u_{2} \rrbracket^{(-)}}{\partial x_{1}}-\frac{\gamma}{2} \mathcal{S}^{(c)} \llbracket p_{1} \rrbracket\left(x_{1}\right)=\alpha \frac{F_{2}}{\pi} \sqrt{\frac{a}{x_{1}}} \frac{1}{x_{1}+a} .
\end{aligned}
$$

The stress intensity factors are then obtained as

$$
K_{\mathrm{I}}=\alpha \sqrt{\frac{2}{\pi a}} F_{2}, \quad K_{\mathrm{II}}=\alpha \sqrt{\frac{2}{\pi a}} F_{1} .
$$

The reader may be surprised by the coupling constant terms in the right-hand side of expressions (C.22), showing a discontinuity of the crack opening at the point of application of the forces. However, these terms can be easily explained by making recourse to the Flamant solution for a half-plane loaded by concentrated forces $F_{1}$ and $F_{2}$ at its surface (Barber, 2002).

Assuming a reference system centred at the point of application of the forces, the surface displacement are determined, up to an arbitrary constant, by

$$
\begin{aligned}
& u_{1}\left(x_{1}\right)=-\frac{F_{1}(1-\nu) \log \left|x_{1}\right|}{\pi \mu}+\frac{F_{2}(1-2 \nu) \operatorname{sign}\left(x_{1}\right)}{4 \mu}, \\
& u_{2}\left(x_{1}\right)=-\frac{F_{1}(1-2 \nu) \operatorname{sign}\left(x_{1}\right)}{4 \mu}-\frac{F_{1}(1-\nu) \log \left|x_{1}\right|}{\pi \mu},
\end{aligned}
$$

where $\mu$ and $\nu$ are the shear modulus and the Poisson's ratio of the half-plane, respectively.

We now apply this solution to the two half-planes making up the cracked bimaterial plane. Of course, we do not expect this solution to match exactly with the correct solution obtained above, since the boundary conditions for the crack problem differ from the Flamant problem along the interface joining the two materials. Nonetheless, let us for the time being make this analogy and calculate from the solution (C.26) what the crack opening would be.

We consider only the coupling (diagonal) terms in the system (C.26), which can explain the observed discontinuity in the crack opening. We also adjust the arbitrary constant in the Flamant solution in order to have the same displacement for the two crack faces at the crack tip. As a result, we obtain

$$
\begin{gathered}
\llbracket u_{1} \rrbracket\left(x_{1}\right)=F_{2} \operatorname{sign}\left(-x_{1}-a\right)\left(\frac{1-2 \nu_{+}}{2 \mu_{+}}+\frac{1-2 \nu_{-}}{2 \mu_{-}}\right)=b \gamma F_{2} \operatorname{sign}\left(-x_{1}-a\right), \\
\llbracket u_{1} \rrbracket\left(x_{1}\right)=-F_{1} \operatorname{sign}\left(-x_{1}-a\right)\left(\frac{1-2 \nu_{+}}{2 \mu_{+}}+\frac{1-2 \nu_{-}}{2 \mu_{-}}\right)=-b \gamma F_{1} \operatorname{sign}\left(-x_{1}-a\right),
\end{gathered}
$$

which is in perfect agreement with our solution (C.22). 


\section{Identities involving convolution integrals}

Theorem D.1. Let $\varphi\left(x_{1}\right)$ be a function defined on $\mathbb{R}$ and possess the derivatives of order $1, \ldots, n$. Assume also that the function $\varphi\left(x_{1}\right)$ is vanishing with all its derivatives as $x_{1} \rightarrow \pm \infty$. Then

$$
\int_{-\infty}^{\infty} \int_{-\infty}^{\infty} \frac{1}{\sqrt{\left(x_{1}-\xi_{1}\right)^{2}+\left(x_{3}-\xi_{3}\right)^{2}}} \frac{\partial^{n} \varphi}{\partial \xi_{1}^{n}} d \xi_{1} d \xi_{3}=-2 \int_{-\infty}^{\infty} \frac{1}{x_{1}-\xi_{1}} \frac{\partial^{n-1} \varphi}{\partial x_{1}^{n-1}} d \xi_{1} .
$$

Proof. Consider the integral

$$
\int_{-R}^{R} \int_{-\infty}^{\infty} \frac{1}{\sqrt{\left(x_{1}-\xi_{1}\right)^{2}+\left(x_{3}-\xi_{3}\right)^{2}}} \frac{\partial^{n} \varphi}{\partial \xi_{1}^{n}} d \xi_{1} d \xi_{3}
$$

and integrate by parts the inner integral, to obtain

$$
\left.\int_{-R}^{R} \frac{1}{\sqrt{\left(x_{1}-\xi_{1}\right)^{2}+\left(x_{3}-\xi_{3}\right)^{2}}} \frac{\partial^{n-1} \varphi}{\partial \xi_{1}^{n-1}}\right|_{\xi_{1}=-\infty} ^{\xi_{1}=\infty} d \xi_{3}-\int_{-R}^{R} \int_{-\infty}^{\infty} \frac{x_{1}-\xi_{1}}{\left[\left(x_{1}-\xi_{1}\right)^{2}+\left(x_{3}-\xi_{3}\right)^{2}\right]^{3 / 2}} \frac{\partial^{n-1} \varphi}{\partial \xi_{1}^{n-1}} d \xi_{1} d \xi_{3} .
$$

The first term in (D.3) is zero and the second term can be rewritten changing the order of integration in the form

$$
-\int_{-\infty}^{\infty}\left(\int_{-R}^{R} \frac{1}{\left[\left(x_{1}-\xi_{1}\right)^{2}+\left(x_{3}-\xi_{3}\right)^{2}\right]^{3 / 2}} d \xi_{3}\right)\left(x_{1}-\xi_{1}\right) \frac{\partial^{n-1} \varphi}{\partial \xi_{1}^{n-1}} d \xi_{1} .
$$

Taking the limit as $R \rightarrow \infty$, the inner integral in (D.4) gives

$$
\lim _{R \rightarrow \infty} \int_{-R}^{R} \frac{1}{\left[\left(x_{1}-\xi_{1}\right)^{2}+\left(x_{3}-\xi_{3}\right)^{2}\right]^{3 / 2}} d \xi_{3}=\frac{2}{\left(x_{1}-\xi_{1}\right)^{2}}
$$

which concludes the proof.

Theorem D.2. Let $\varphi\left(x_{1}, x_{3}\right)$ be a function defined on $\mathbb{R}^{2}$ and possess the Fourier transform with respect to $x_{1}$ and $x_{3}$, denoted by $\bar{\varphi}(\beta, \lambda)$. Then

$$
\begin{array}{r}
\int_{-\infty}^{\infty} \int_{-\infty}^{\infty} \frac{x_{1}-\xi_{1}}{\left(x_{1}-\xi_{1}\right)^{2}+\left(x_{3}-\xi_{3}\right)^{2}} \frac{\partial \varphi}{\partial \xi_{1}} d \xi_{1} d \xi_{3}+\int_{-\infty}^{\infty} \int_{-\infty}^{\infty} \frac{x_{3}-\xi_{3}}{\left(x_{1}-\xi_{1}\right)^{2}+\left(x_{3}-\xi_{3}\right)^{2}} \frac{\partial \varphi}{\partial \xi_{3}} d \xi_{1} d \xi_{3}= \\
=2 \pi \varphi\left(x_{1}, x_{3}\right) .
\end{array}
$$

Proof. Consider the Fourier transform $\bar{\varphi}(\beta, \lambda)$ and write

$$
\bar{\varphi}(\beta, \lambda)=\frac{\beta^{2}}{\beta^{2}+\lambda^{2}} \cdot \bar{\varphi}(\beta, \lambda)+\frac{\lambda^{2}}{\beta^{2}+\lambda^{2}} \cdot \bar{\varphi}(\beta, \lambda) .
$$

To conclude the proof, apply the inversion Fourier transform to both side of (D.7) and use the first two formulae given in Tab. 1 .

Theorem D.3. Let $\varphi\left(x_{1}, x_{3}\right)$ be a function defined on $\mathbb{R}^{2}$ and possess the Fourier transform with respect $x_{1}$ and $x_{3}$, denoted by $\bar{\varphi}(\beta, \lambda)$. Then

$$
\int_{-\infty}^{\infty} \int_{-\infty}^{\infty} \frac{x_{3}-\xi_{3}}{\left(x_{1}-\xi_{1}\right)^{2}+\left(x_{3}-\xi_{3}\right)^{2}} \frac{\partial \varphi}{\partial \xi_{1}} d \xi_{1} d \xi_{3}=\int_{-\infty}^{\infty} \int_{-\infty}^{\infty} \frac{x_{1}-\xi_{1}}{\left(x_{1}-\xi_{1}\right)^{2}+\left(x_{3}-\xi_{3}\right)^{2}} \frac{\partial \varphi}{\partial \xi_{3}} d \xi_{1} d \xi_{3} .
$$

Proof. Consider the Fourier transform $\bar{\varphi}(\beta, \lambda)$ and write

$$
\frac{\beta}{\beta^{2}+\lambda^{2}} \cdot \lambda \bar{\varphi}(\beta, \lambda)=\frac{\lambda}{\beta^{2}+\lambda^{2}} \cdot \beta \bar{\varphi}(\beta, \lambda)
$$

To conclude the proof, apply the inversion Fourier transform to both side of (D.7) and use the third and fourth formulae in Tab. 1 


\section{E Double Fourier transform inversion for the 3D case}

The following four integrals will be used in the analysis (Gradshteyn and Ryzhik, 1965):

$$
\begin{gathered}
\int_{0}^{\infty} \frac{\cos a x}{\sqrt{\beta^{2}+x^{2}}} d x=K_{0}(a \beta), \quad a>0, \quad \operatorname{Re} \beta>0, \\
\int_{0}^{\infty} \frac{x \sin a x}{\beta^{2}+x^{2}} d x=\frac{\pi}{2} e^{-a \beta}, \quad a>0, \quad \operatorname{Re} \beta>0, \\
\int_{0}^{\infty} K_{0}(a \beta) \cos (b \beta) d \beta=\frac{\pi}{2} \frac{1}{\sqrt{a^{2}+b^{2}}}, \quad \operatorname{Re} a>0, \\
\int_{0}^{\infty} e^{-a \beta} \cos (b \beta) d \beta=\frac{a}{a^{2}+b^{2}}, \quad a>0,
\end{gathered}
$$

where $K_{0}(z)$ is the modified Bessel function of the second kind of zero order, satisfying the differential equation

$$
z^{2} y^{\prime \prime}+z y^{\prime}-z^{2} y=0 .
$$

In some sense, the last two integrals are the Fourier inversion formulae for the first two.

We also shall make use of the fact that the Fourier transform of the convolution is given by the product of the Fourier transforms, which leads to

$$
\mathcal{F}_{\beta}^{-1} \mathcal{F}_{\lambda}^{-1}[\bar{g}(\beta, \lambda) \cdot \bar{f}(\beta, \lambda)]=\int_{-\infty}^{\infty} \int_{-\infty}^{\infty} g\left(x_{1}-\xi_{1}, x_{3}-\xi_{3}\right) f\left(\xi_{1}, \xi_{3}\right) d \xi_{1} d \xi_{3} .
$$

It is evident what to do with the factors $\beta$ and $\lambda$. Namely, these factors arise when taking the Fourier transform of the derivatives instead of the function itself:

$$
\beta \bar{f}(\beta, \lambda)=i \overline{\frac{\partial f}{\partial x_{1}}}(\beta, \lambda), \quad \lambda \bar{f}(\beta, \lambda)=i \overline{\frac{\partial f}{\partial x_{3}}}(\beta, \lambda) .
$$

Analogous identities can be written for the factors $\beta^{2}, \lambda^{2}$ and $\beta \lambda$ involving higher order derivatives:

$$
\beta^{2} \bar{f}(\beta, \lambda)=-\frac{\overline{\partial^{2} f}}{\partial x_{1}^{2}}(\beta, \lambda), \quad \lambda^{2} \bar{f}(\beta, \lambda)=-\frac{\overline{\partial^{2} f}}{\partial x_{3}^{2}}(\beta, \lambda), \quad \beta \lambda \bar{f}(\beta, \lambda)=-\overline{\frac{\partial^{2} f}{\partial x_{1} \partial x_{3}}}(\beta, \lambda) .
$$

Taking into account the following identity:

$$
\sqrt{\beta^{2}+\lambda^{2}}=\frac{\beta^{2}+\lambda^{2}}{\sqrt{\beta^{2}+\lambda^{2}}}
$$

we need to evaluate only the following three basic integrals:

$$
\begin{aligned}
\mathcal{F}_{\beta}^{-1} \mathcal{F}_{\lambda}^{-1}\left[\frac{1}{\sqrt{\beta^{2}+\lambda^{2}}}\right] & =\frac{1}{4 \pi^{2}} \int_{-\infty}^{\infty} \int_{-\infty}^{\infty} \frac{1}{\sqrt{\beta^{2}+\lambda^{2}}} e^{-i x_{1} \beta} e^{-i x_{3} \lambda} d \beta d \lambda, \\
\mathcal{F}_{\beta}^{-1} \mathcal{F}_{\lambda}^{-1}\left[\frac{\lambda}{\beta^{2}+\lambda^{2}}\right] & =\frac{1}{4 \pi^{2}} \int_{-\infty}^{\infty} \int_{-\infty}^{\infty} \frac{\lambda}{\beta^{2}+\lambda^{2}} e^{-i x_{1} \beta} e^{-i x_{3} \lambda} d \beta d \lambda, \\
\mathcal{F}_{\beta}^{-1} \mathcal{F}_{\lambda}^{-1}\left[\frac{\beta}{\beta^{2}+\lambda^{2}}\right] & =\frac{1}{4 \pi^{2}} \int_{-\infty}^{\infty} \int_{-\infty}^{\infty} \frac{\beta}{\beta^{2}+\lambda^{2}} e^{-i x_{1} \beta} e^{-i x_{3} \lambda} d \beta d \lambda .
\end{aligned}
$$

Let us consider the first integral (E.10). By (E.1) we obtain

$$
\int_{-\infty}^{\infty} e^{-i x_{3} \lambda} \frac{d \lambda}{\sqrt{\beta^{2}+\lambda^{2}}}=2 \int_{0}^{\infty} \frac{\cos \left(\left|x_{3}\right| \lambda\right)}{\sqrt{\beta^{2}+\lambda^{2}}} d \lambda=2 \begin{cases}K_{0}\left(\left|x_{3}\right| \beta\right), & \operatorname{Re} \beta>0 \\ K_{0}\left(-\left|x_{3}\right| \beta\right), & -\operatorname{Re} \beta>0 .\end{cases}
$$


Making recourse to (E.3), we conclude that

$$
\begin{aligned}
\mathcal{F}_{\beta}^{-1} \mathcal{F}_{\lambda}^{-1}\left[\frac{1}{\sqrt{\beta^{2}+\lambda^{2}}}\right] & =\frac{1}{2 \pi^{2}} \int_{-\infty}^{\infty} e^{-i x_{1} \beta} K_{0}\left(\left|x_{3}\right||\beta|\right) d \beta, \\
& =\frac{1}{\pi^{2}} \int_{0}^{\infty} K_{0}\left(\left|x_{3}\right| \beta\right) \cos \left(x_{1} \beta\right) d \beta, \\
& =\frac{1}{2 \pi} \frac{1}{\sqrt{x_{1}^{2}+x_{3}^{2}}} .
\end{aligned}
$$

We note the the function transforms into itself (multiplied by a constant factor) after double Fourier transform.

Let us now consider the second integral (E.11). By means of (E.2) we write

$$
\int_{-\infty}^{\infty} e^{-i x_{3} \lambda} \frac{\lambda d \lambda}{\beta^{2}+\lambda^{2}}=-2 i \operatorname{sign}\left(x_{3}\right) \int_{0}^{\infty} \frac{\lambda \sin \left(\left|x_{3}\right| \lambda\right)}{\beta^{2}+\lambda^{2}} d \lambda=-\pi i \operatorname{sign}\left(x_{3}\right) \begin{cases}e^{-\left|x_{3}\right| \beta}, & \operatorname{Re} \beta>0, \\ e^{\left|x_{3}\right| \beta}, & -\operatorname{Re} \beta>0 .\end{cases}
$$

We can now use the formula (E.4) to obtain

$$
\begin{aligned}
\mathcal{F}_{\beta}^{-1} \mathcal{F}_{\lambda}^{-1}\left[\frac{\lambda}{\beta^{2}+\lambda^{2}}\right] & =\frac{-i \operatorname{sign}\left(x_{3}\right)}{4 \pi} \int_{-\infty}^{\infty} e^{-i x_{1} \beta} e^{-\left|x_{3}\right||\beta|} d \beta, \\
& =\frac{-i \operatorname{sign}\left(x_{3}\right)}{2 \pi} \int_{0}^{\infty} e^{-\left|x_{3}\right| \beta} \cos \left(x_{1} \beta\right) d \beta, \\
& =\frac{-i \operatorname{sign}\left(x_{3}\right)}{2 \pi} \frac{\left|x_{3}\right|}{x_{1}^{2}+x_{3}^{2}}=\frac{-i}{2 \pi} \frac{x_{3}}{x_{1}^{2}+x_{3}^{2}} .
\end{aligned}
$$

Again, the function transforms into itself (multiplied by a constant factor) after double Fourier transform.

The last integral (E.12) can be evaluated in a similar way and we have

$$
\mathcal{F}_{\beta}^{-1} \mathcal{F}_{\lambda}^{-1}\left[\frac{\beta}{\beta^{2}+\lambda^{2}}\right]=\frac{-i}{2 \pi} \frac{x_{1}}{x_{1}^{2}+x_{3}^{2}} .
$$

Equations (E.14), (E.16) and (E.17) are the basic integrals we use to perform the double Fourier inversion

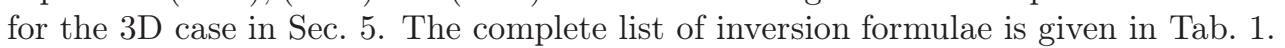


Function: Double Fourier inversion:

\begin{tabular}{ll}
$\bar{f}(\beta, \lambda)$ & $\mathcal{F}_{\beta}^{-1} \mathcal{F}_{\lambda}^{-1}[\bar{f}(\beta, \lambda)]$ \\
\hline$\frac{\beta}{\beta^{2}+\lambda^{2}} \cdot \beta \bar{\varphi}$ & $\frac{1}{2 \pi} \frac{x_{1}}{x_{1}^{2}+x_{3}^{2}} \circledast \frac{\partial \varphi}{\partial x_{1}}$ \\
$\frac{\lambda}{\beta^{2}+\lambda^{2}} \cdot \lambda \bar{\varphi}$ & $\frac{1}{2 \pi} \frac{x_{3}}{x_{1}^{2}+x_{3}^{2}} \circledast \frac{\partial \varphi}{\partial x_{3}}$ \\
$\frac{\beta}{\beta^{2}+\lambda^{2}} \cdot \lambda \bar{\varphi}$ & $\frac{1}{2 \pi} \frac{x_{1}}{x_{1}^{2}+x_{3}^{2}} \circledast \frac{\partial \varphi}{\partial x_{3}}$ \\
$\frac{\lambda}{\beta^{2}+\lambda^{2}} \cdot \beta \bar{\varphi}$ & $\frac{1}{2 \pi} \frac{x_{3}}{x_{1}^{2}+x_{3}^{2}} \circledast \frac{\partial \varphi}{\partial x_{1}}$ \\
$\frac{1}{\sqrt{\beta^{2}+\lambda^{2}}} \cdot \beta \bar{\varphi}$ & $\frac{i}{2 \pi} \frac{1}{\sqrt{x_{1}^{2}+x_{3}^{2}}} \circledast \frac{\partial \varphi}{\partial x_{1}}$ \\
$\frac{1}{\sqrt{\beta^{2}+\lambda^{2}}} \cdot \lambda \bar{\varphi}$ & $\frac{i}{2 \pi} \frac{1}{\sqrt{x_{1}^{2}+x_{3}^{2}} \circledast \frac{\partial \varphi}{\partial x_{3}}}$ \\
$\frac{1}{\sqrt{\beta^{2}+\lambda^{2}}} \cdot \beta^{2} \bar{\varphi}$ & $-\frac{1}{2 \pi} \frac{1}{\sqrt{x_{1}^{2}+x_{3}^{2}} \circledast \frac{\partial^{2} \varphi}{\partial x_{1}^{2}}}$ \\
$\frac{1}{\sqrt{\beta^{2}+\lambda^{2}}} \cdot \lambda^{2} \bar{\varphi}$ & $-\frac{1}{2 \pi} \frac{1}{\sqrt{x_{1}^{2}+x_{3}^{2}} \circledast \frac{\partial^{2} \varphi}{\partial x_{3}^{2}}}$ \\
$\frac{1}{\sqrt{\beta^{2}+\lambda^{2}}} \cdot \beta \lambda \bar{\varphi}$ & $-\frac{1}{2 \pi} \frac{1}{\sqrt{x_{1}^{2}+x_{3}^{2}} \circledast \frac{\partial^{2} \varphi}{\partial x_{1} \partial x_{3}}}$ \\
$\frac{\sqrt{\beta^{2}+\lambda^{2}} \cdot \bar{\varphi}}{\frac{1}{2 \pi} \frac{1}{\sqrt{x_{1}^{2}+x_{3}^{2}}} \circledast\left(\frac{\partial^{2} \varphi}{\partial x_{1}^{2}}+\frac{\partial^{2} \varphi}{\partial x_{3}^{2}}\right)}$
\end{tabular}

Table 1: Double Fourier inversion formulae 